\title{
GALOIS-THEORETIC CHARACTERIZATION OF ISOMORPHISM CLASSES OF MONODROMICALLY FULL HYPERBOLIC CURVES OF GENUS ZERO
}

\author{
YUICHIRO HOSHI
}

\begin{abstract}
Let $l$ be a prime number. In this paper, we prove that the isomorphism class of an $l$-monodromically full hyperbolic curve of genus zero over a finitely generated extension of the field of rational numbers is completely determined by the kernel of the natural pro-l outer Galois representation associated to the hyperbolic curve. This result can be regarded as a genus zero analogue of a result due to Mochizuki which asserts that the isomorphism class of an elliptic curve which does not admit complex multiplication over a number field is completely determined by the kernels of the natural Galois representations on the various finite quotients of its Tate module.
\end{abstract}

\section{Contents}

Introduction . . . . . . . . . . . . . . . . . . . . . . . . . 48

0. Notation and conventions . . . . . . . . . . . . . . . . . . . . 52

1. Outer monodromy representations . . . . . . . . . . . . . . . . 53

2. Monodromically full points and curves . . . . . . . . . . . . . . . . . 60

3. Relationship between monodromic fullness and certain properties of hyperbolic

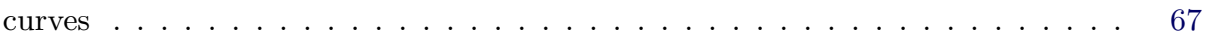

4. Moduli stacks of hyperbolic curves of genus zero . . . . . . . . . . . . . . . 72

5. A Grothendieck conjecture-type lemma for certain images of the universal monodromy . . . . . . . . . . . . . . . . . 76

6. Proof of the main result . . . . . . . . . . . . . . . . . . . . 81

7. Example I: Hyperbolic curves of type $(0,4)$ over number fields . . . . . . . . . . 83

8. Example II: Nonisotrivial hyperbolic curves of type $(0,4) \ldots \ldots$. . . . . . . . 93

Appendix. Ramification of outer Galois representations and isomorphism classes of hyperbolic curves . . . . . . . . . . . . . . . . . . . . 96

Acknowledgments . . . . . . . . . . . . . . . . . . . . 99

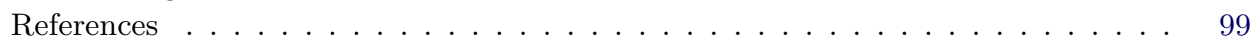

Received December 24, 2009. Revised November 17, 2010. Accepted December 2, 2010. 2010 Mathematics Subject Classification. Primary 14H30; Secondary 14H10.

The author's research was supported by Grant-in-Aid for Young Scientists (B) 20740010, Japan Society for the Promotion of Science.

(C) 2011 by The Editorial Board of the Nagoya Mathematical Journal 


\section{Introduction}

Throughout this article, let $k$ be a field of characteristic zero, let $\bar{k}$ be an algebraic closure of $k$, and let $G_{k} \stackrel{\text { def }}{=} \operatorname{Gal}(\bar{k} / k)$. We prove that if $l$ is a prime number, then the isomorphism class of an $l$-monodromically full hyperbolic curve of genus zero over a finitely generated extension of the field of rational numbers is completely determined by the kernel of the associated pro- $l$ outer Galois representation.

Mochizuki proved the following theorem (see [14, Theorem 1.1]).

Let $\left(E_{1}, o_{1} \in E_{1}(k)\right),\left(E_{2}, o_{2} \in E_{2}(k)\right)$ be elliptic curves over $k$ which do not admit complex multiplication over $\bar{k}$. Suppose that $k$ is a number field, that is, a finite extension of the field of rational numbers. Then the following conditions are equivalent:

(i) $\left(E_{1}, o_{1}\right)$ is isomorphic to $\left(E_{2}, o_{2}\right)$ over $k$;

(ii) for $i=1,2$, write $T\left(E_{i}, o_{i}\right)$ for the full Tate module of $\left(E_{i}, o_{i}\right)$ and write

$$
\rho_{\left(E_{i}, o_{i}\right) / k}^{(n)}: G_{k} \longrightarrow \operatorname{Aut}\left(T\left(E_{i}, o_{i}\right) \otimes_{\widehat{\mathbb{Z}}}(\mathbb{Z} / n \mathbb{Z})\right)
$$

for the natural Galois representation on $T\left(E_{i}, o_{i}\right) \otimes_{\widehat{\mathbb{Z}}}(\mathbb{Z} / n \mathbb{Z})$; then $\operatorname{Ker}\left(\rho_{\left(E_{1}, o_{1}\right) / k}^{(n)}\right)=\operatorname{Ker}\left(\rho_{\left(E_{2}, o_{2}\right) / k}^{(n)}\right)$ for any positive integer $n$.

In this paper, we prove a genus zero analogue of the above result of Mochizuki. The main theorem presented here is the following (see Theorem 6.1).

TheOREM A (Galois-theoretic characterization of isomorphism classes of monodromically full hyperbolic curves of genus zero). Let $l$ be a prime number, let $k$ be a finitely generated field of characteristic zero (i.e., a finitely generated extension of the field of rational numbers), and let $X_{1}=\left(C_{1}\right.$, $\left.D_{1} \subseteq C_{1}\right), X_{2}=\left(C_{2}, D_{2} \subseteq C_{2}\right)$ be hyperbolic curves (see Definition 1.1(ii)) of genus zero over $k$ which are l-monodromically full (see Definition 2.2(i)). Suppose that the following condition $(\dagger)^{\text {prime }}$ is satisfied.

$(\dagger)^{\text {prime }}$ : There exists a finite Galois extension $k^{\prime} \subseteq \bar{k}$ of $k$ of degree prime to

$l$ such that $X_{1} \otimes_{k} k^{\prime}$ and $X_{2} \otimes_{k} k^{\prime}$ are split (see Definition 1.5(i)).

(For example, if one of the following conditions is satisfied, then the above condition $(\dagger)^{\text {prime }}$ is satisfied:

- $X_{1}$ and $X_{2}$ are split;

- if we write $r_{i}$ for the number of cusps of $X_{i}$, that is, if $X_{i}$ is of type $\left(0, r_{i}\right)$,

then $l$ is prime to $r_{1}$ ! and $r_{2}$ ! or, equivalently, $r_{1}, r_{2}<l$.)

Then the following conditions are equivalent: 
(i) $X_{1}$ is isomorphic to $X_{2}$ over $k$;

(ii) for $i=1,2$, write

$$
\rho_{X_{i} / k}^{\{l\}}: G_{k} \longrightarrow \operatorname{Out}\left(\pi_{1}\left(\left(C_{i} \backslash D_{i}\right) \otimes_{k} \bar{k}\right)^{(l)}\right)
$$

for the natural pro-l outer Galois representation associated to $X_{i}$, then $\operatorname{Ker}\left(\rho_{X_{1} / k}^{\{l\}}\right)=\operatorname{Ker}\left(\rho_{X_{2} / k}^{\{l\}}\right)$.

Roughly speaking, Theorem A asserts that the isomorphism class of an $l$-monodromically full hyperbolic curve of genus zero over a finitely generated field of characteristic zero which satisfies the condition $(\dagger)^{\text {prime }}$ is completely determined by the kernel of the associated pro- $l$ outer Galois representation. Note that the fact that the isomorphism class of a hyperbolic curve over a generalized sub-l-adic field (see the discussion on numbers in Section 0) is completely determined by the associated pro- $l$ outer Galois representation was proved in [14, Theorem 4.12].

Although we introduce the term l-monodromically full in this paper, the corresponding notion was studied by Matsumoto and Tamagawa in [11]. It is known (see [11, Theorem 1.2], as well as Corollary 2.6 below) that many hyperbolic curves are $l$-monodromically full. This property of being $l$-monodromically full may be regarded as an analogue for hyperbolic curves of the property of not admitting complex multiplication for elliptic curves. In fact, if a hyperbolic curve $X$ of type $(g, r)$ over a finitely generated extension $k$ of the field of rational numbers is $l$-monodromically full, then the following hold:

- $X$ has no special symmetry (i.e., roughly speaking, the automorphism group of $X$ over $\bar{k}$ is isomorphic to the automorphism group of a general hyperbolic curve of type $(g, r)$ over $\bar{k}$; see Definition 3.3, Proposition 3.4); in particular, if $2 g-2+r \geq 3$, then there is no nontrivial automorphism of $X$ over $\bar{k}$;

- $X$ is of $\{l\}$-AIJ-type, where AIJ stands for absolutely irreducibe Jacobian (i.e., roughly speaking, the $l$-adic Tate module of the Jacobian variety of the compactification of $X$ is, as a Galois module, absolutely irreducible; see Definition 3.5, Proposition 3.6);

- $X$ does not have a JCM-component, where JCM stands for Jacobian complex multiplication (i.e., roughly speaking, there is no subabelian variety with complex multiplication over $\bar{k}$ of the Jacobian variety of the compactification of $X$; see Definition 3.7, Proposition 3.8). 
In this paper, we consider as an example the monodromic fullness of hyperbolic curves of type $(0,4)$, and we obtain results concerning sufficient conditions for such a hyperbolic curve to be monodromically full (see Theorem 7.9 and Corollaries 7.11, 7.12, and 8.2). These results, together with Theorem A, imply the following result (see Corollaries 7.13 and 8.3).

THEOREM B (Galois-theoretic characterization of isomorphism classes of certain hyperbolic curves of type $(0,4))$. Let $k$ be a finitely generated field of characteristic zero, that is, a finitely generated extension of the field of rational numbers, and let $X_{1}=\left(C_{1}, D_{1} \subseteq C_{1}\right), X_{2}=\left(C_{2}, D_{2} \subseteq C_{2}\right)$ be hyperbolic curves (see Definition 1.1(ii)) of type $(0,4)$ over $k$. Suppose that one of the following conditions is satisfied.

- The field $k$ is a number field, that is, a finite extension of the field of rational numbers, and moreover, if we write $\mathfrak{o}_{k}^{*}$ for the group of units of the ring of integers $\mathfrak{o}_{\bar{k}}$ of $\bar{k}$, then $\mathfrak{m}_{X_{1}} \cap \mathfrak{o}_{\bar{k}}^{*}=\mathfrak{m}_{X_{2}} \cap \mathfrak{o}_{\bar{k}}^{*}=\emptyset$ (see Definition 7.10).

- The hyperbolic curves $X_{1}$ and $X_{2}$ are not NF-isotrivial, where NF stands for number field (see Definition 8.1).

Then the following conditions are equivalent:

(i) $X_{1}$ is isomorphic to $X_{2}$ over $k$;

(ii) there exists an infinite set $\Sigma$ of prime numbers such that, for any $l \in \Sigma$, if we write

$$
\rho_{X_{i} / k}^{\{l\}}: G_{k} \longrightarrow \operatorname{Out}\left(\pi_{1}\left(\left(C_{i} \backslash D_{i}\right) \otimes_{k} \bar{k}\right)^{(l)}\right)
$$

for the natural pro-l outer Galois representation associated to $X_{i}$, then $\operatorname{Ker}\left(\rho_{X_{1} / k}^{\{l\}}\right)=\operatorname{Ker}\left(\rho_{X_{2} / k}^{\{l\}}\right)$.

On the other hand, one may also take the point of view that Theorems $\mathrm{A}$ and B serve to highlight the difference between the profinite and pro-l outer Galois representations associated to a hyperbolic curve. In [11], Matsumoto and Tamagawa compared the profinite and pro- $l$ outer Galois representations associated to hyperbolic curves. One result obtained in [11] which shows the difference between the profinite and pro- $l$ outer Galois representations is found in my following summary.

Summary: The image of the profinite outer Galois representation associated to any hyperbolic curve of type $(g, r)$ over a number field $k$ has trivial intersection with the image of the outer profinite geometric universal monodromy representation of $\pi_{1}\left(\mathcal{M}_{g, r} \otimes_{k} \bar{k}\right)$ (see [11, Theorem 1.1] and [8, Corollary 6.4]). On 
the other hand, there exist many hyperbolic curves of type $(g, r)$ over number fields $k$ for which the image of the associated pro- $l$ outer Galois representation contains the image of the outer pro- $l$ geometric universal monodromy representation of $\pi_{1}\left(\mathcal{M}_{g, r} \otimes_{k} \bar{k}\right)$ (see [11, Theorem 1.2]).

By Theorems A and B (and Theorem C below), one obtains another result which highlights the difference between the profinite and pro-l outer Galois representations, as I show in this summary.

\begin{abstract}
Summary: The kernel of the profinite outer Galois representation associated to any hyperbolic curve over a number field is always trivial; namely, the kernel does not depend on the given hyperbolic curve (see [8, Theorem C]). On the other hand, the kernel of the pro- $l$ outer Galois representation associated to a hyperbolic curve over a number field depends strongly on the given hyperbolic curve (see Theorems A and B; see also Theorem C below).
\end{abstract}

Finally, in the appendix, we prove the following finiteness result, which is related to the main result of this paper (see Corollary A.4).

Theorem C (finiteness of the set of isomorphism classes of certain hyperbolic curves). Let $l$ be a prime number, let $k$ be a number field, that is, a finite extension of the field of rational numbers, let $(g, r)$ be a pair of nonnegative integers such that $2 g-2+r>0$, and let $N \subseteq G_{k}$ be a normal closed subgroup of $G_{k}$. Then there are only finitely many isomorphism classes over $k$ of hyperbolic curves $X$ of type $(g, r)$ over $k$ for which the kernel of the natural pro-l outer Galois representation associated to $X$ coincides with $N$.

This result follows immediately from various well-known finiteness theorems in number theory and arithmetic geometry, together with Oda-Tamagawa's criterion for good reduction of hyperbolic curves. It seems to the author that this result is likely to be well known. However, since this result could not be found in the literature, the author has decided to give a proof of it in the appendix of this paper.

This article is organized as follows. In Section 1, we review some generalities concerning outer monodromy representations arising from hyperbolic curves. In Section 2, we define the notion of a $\Sigma$-monodromically full hyperbolic curve, as well as the related notion of a $\Sigma$-monodromically full point. In Section 3, we consider the relationship between monodromic fullness and certain properties of hyperbolic curves. In Section 4, we consider the moduli stacks of hyperbolic curves of genus zero. In Section 5, we prove a Grothendieck conjecture-type lemma for certain images of the universal 
monodromy. In Section 6, we derive Theorem A from the results obtained in Sections 4 and 5. In Sections 7 and 8, we consider the monodromic fullness of hyperbolic curves of type $(0,4)$. In particular, we obtain results concerning sufficient conditions for such a hyperbolic curve to be monodromically full, and we prove Theorem B. In the appendix, we derive Theorem C as a consequence of various well-known finiteness theorems in number theory and arithmetic geometry, together with Oda-Tamagawa's criterion for good reduction of hyperbolic curves.

\section{$\S 0$. Notation and conventions}

Numbers: A finite extension (resp., finitely generated extension) of the field of rational numbers will be referred to as a number field (resp., finitely generated field of characteristic zero). If $p$ is a prime number, then a field which may be embedded as a subfield of a finitely generated extension of the field of fractions of the ring of Witt vectors with coefficients in an algebraic closure of the finite field of $p$ elements will be referred to as a generalized sub-p-adic field (see [14, Definition 4.11]).

Topological groups: Let $G$ be a topological group, and let $\mathbf{P}$ be a property for a topological group (e.g., abelian or pro- $l$ for some prime number $l$ ). Then we say that $G$ is almost $\mathbf{P}$ if there exists an open subgroup of $G$ that is $\mathbf{P}$.

If $G$ is a topological group, then we write $G^{\text {ab }}$ for the abelianization of $G$, that is, the quotient of $G$ by the closure of the commutator subgroup of $G$.

If $G$ is a topological group and if $H \subseteq G$ is a closed subgroup of $G$, then we write $Z_{G}(H)$ for the centralizer of $H$ in $G$, that is,

$$
Z_{G}(H) \stackrel{\text { def }}{=}\left\{g \in G \mid g h g^{-1}=h \text { for any } h \in H\right\} \subseteq G,
$$

we write $Z_{G}^{\text {loc }}(H)$ for the local centralizer of $H$ in $G$, that is,

$$
Z_{G}^{\mathrm{loc}}(H) \stackrel{\text { def }}{=} \underset{H^{\prime} \subseteq H}{\lim _{\longrightarrow}} Z_{G}\left(H^{\prime}\right) \subseteq G,
$$

where $H^{\prime} \subseteq H$ ranges over the open subgroups of $H$, we write $Z(G) \stackrel{\text { def }}{=}$ $Z_{G}(G)$ for the center of $G$, and we write $Z^{\text {loc }}(G) \stackrel{\text { def }}{=} Z_{G}^{\text {loc }}(G)$ for the local center of $G$. It is immediate from the various definitions involved that $Z_{G}(H) \subseteq Z_{G}^{\text {loc }}(H)$ and that if $H_{1}, H_{2} \subseteq G$ are closed subgroups of $G$ such that $H_{1} \subseteq H_{2}$ (resp., $H_{1} \subseteq H_{2} ; H_{1} \cap H_{2}$ is open in $H_{1}$ and $H_{2}$ ), then $Z_{G}\left(H_{2}\right) \subseteq Z_{G}\left(H_{1}\right)$ (resp., $\left.Z_{G}^{\mathrm{loc}}\left(H_{2}\right) \subseteq Z_{G}^{\mathrm{loc}}\left(H_{1}\right) ; Z_{G}^{\mathrm{loc}}\left(H_{1}\right)=Z_{G}^{\mathrm{loc}}\left(H_{2}\right)\right)$. 
We say that a topological group $G$ is center-free (resp., slim) if $Z(G)=$ $\{1\}$ (resp., $Z^{\mathrm{loc}}(G)=\{1\}$ ). Note that it follows from [15, Remark 0.1.3] that a profinite group $G$ is slim if and only if every open subgroup of $G$ has trivial center.

If $G$ is a topological group, then we denote the group of (continuous) automorphisms of $G$ by $\operatorname{Aut}(G)$, and we denote the group of inner automorphisms of $G$ by $\operatorname{Inn}(G) \subseteq \operatorname{Aut}(G)$. The conjugation by elements of $G$ determines a surjection $G \rightarrow \operatorname{Inn}(G)$. Thus, we have a homomorphism $G \rightarrow \operatorname{Aut}(G)$ whose image is $\operatorname{Inn}(G) \subseteq \operatorname{Aut}(G)$. We denote by $\operatorname{Out}(G)$ the quotient of $\operatorname{Aut}(G)$ by the normal subgroup $\operatorname{Inn}(G) \subseteq \operatorname{Aut}(G)$. In particular, if $G$ is center-free, then the natural homomorphism $G \rightarrow \operatorname{Inn}(G)$ is an isomorphism; thus, we have an exact sequence of groups

$$
1 \longrightarrow G \longrightarrow \operatorname{Aut}(G) \longrightarrow \operatorname{Out}(G) \longrightarrow 1 \text {. }
$$

Moreover, if $G$ is profinite and topologically finitely generated, then one easily verifies that the topology of $G$ admits a basis of characteristic open subgroups, which thus induces a profinite topology on $\operatorname{Aut}(G)$, hence also on Out $(G)$, with respect to which the above exact sequence determines an exact sequence of profinite groups. If $J$ is a profinite group and if $\rho: J \rightarrow \operatorname{Out}(G)$ is a continuous homomorphism, then we denote by $G \stackrel{\text { out }}{\rtimes} J$ the profinite group obtained by pulling back the above exact sequence of profinite groups via $\rho$. Thus, we have a natural exact sequence of profinite groups

$$
1 \longrightarrow G \longrightarrow G \stackrel{\text { out }}{\rtimes} J \longrightarrow J \longrightarrow 1
$$

\section{$\S 1$. Outer monodromy representations}

Throughout this article, let $k$ be a field of characteristic zero, and let $\bar{k}$ be an algebraic closure of $k$. If $k^{\prime} \subseteq \bar{k}$ is an algebraic (possibly infinite) extension of $k$, then we write $G_{k^{\prime}} \stackrel{\text { def }}{=} \operatorname{Gal}\left(\bar{k} / k^{\prime}\right)$.

In this section, we review some generalities concerning outer monodromy representations arising from hyperbolic curves. Here, let $(g, r)$ be a pair of nonnegative integers such that $2 g-2+r>0$, and let $\Sigma$ be a nonempty set of prime numbers.

Definition 1.1. Let $S$ be a scheme. We have the following.

(i) Let $C$ be a scheme over $S$, and let $s_{i}: S \rightarrow C$ be a section of the structure morphism of $C$, where $i=1, \ldots, r$. Then we say that $\left(C,\left(s_{1}, \ldots, s_{r}\right)\right)$ 
is an r-pointed smooth curve of genus $g$ over $S$ whose marked points are equipped with an ordering if $C$ is smooth and proper over $S$, if any geometric fiber of $C \rightarrow S$ is a (necessarily smooth and proper) connected curve of genus $g$, and if the image of $s_{i}$ does not intersect the image of $s_{j}$ if $i \neq j$.

(ii) Let $C$ be a scheme over $S$, and let $D \subseteq C$ be a closed subscheme of $C$. Then we say that $(C, D \subseteq C)$ is a hyperbolic curve of type $(g, r)$ over $S$ if $C$ is smooth and proper over $S$, if any geometric fiber of $C \rightarrow S$ is a (necessarily smooth and proper) connected curve of genus $g$, and if the composite $D \hookrightarrow C \rightarrow S$ is a finite étale covering over $S$ of degree $r$.

Definition 1.2. We have the following.

(i) We denote by $\mathcal{M}_{g, r} \rightarrow$ Spec $k$ the moduli stack (see [5], [10]) of $r$ pointed smooth curves of genus $g$ over $k$-schemes whose marked points are equipped with orderings (see Definition 1.1(i)), and we denote by $\left(\mathcal{C}_{g, r} \rightarrow \mathcal{M}_{g, r},\left(s_{1}^{\mathcal{M}}, \ldots, s_{r}^{\mathcal{M}}\right)\right)$ the universal curve over $\mathcal{M}_{g, r}$.

(ii) We denote by $\mathcal{M}_{g,[r]} \rightarrow$ Spec $k$ the moduli stack of hyperbolic curves of type $(g, r)$ over $k$-schemes (see Definition 1.1(ii)), and we denote by $\left(\mathcal{C}_{g,[r]} \rightarrow \mathcal{M}_{g,[r]}, D_{g,[r]}^{\mathcal{M}} \subseteq \mathcal{C}_{g,[r]}\right)$ the universal curve over $\mathcal{M}_{g,[r]}$.

It follows from the various definitions involved that we have a commutative diagram

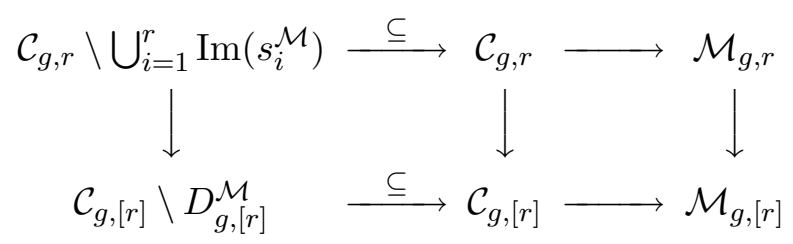

such that the two squares in this diagram are Cartesian. Moreover, it is well known that in this commutative diagram, the right-hand vertical arrow $\mathcal{M}_{g, r} \rightarrow \mathcal{M}_{g,[r]}$ is a finite étale Galois covering whose Galois group is isomorphic to the symmetric group on $r$ letters $\mathfrak{S}_{r}$. In particular, we obtain a commutative diagram 


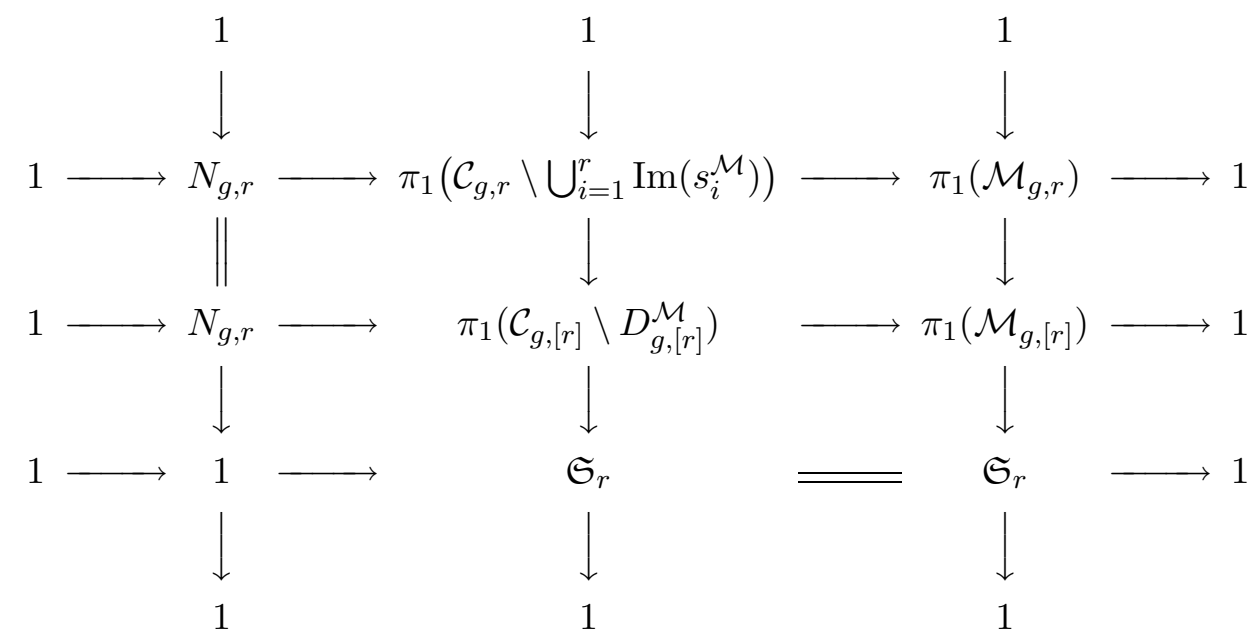

where $N_{g, r}$ is the kernel of the surjection $\pi_{1}\left(\mathcal{C}_{g, r} \backslash \bigcup_{i=1}^{r} \operatorname{Im}\left(s_{i}^{\mathcal{M}}\right)\right) \rightarrow \pi_{1}\left(\mathcal{M}_{g, r}\right)$ and where the vertical and horizontal sequences are exact. (See [20] for the fundamental groups of stacks.)

Definition 1.3. We have the following.

(i) We write

$$
\Delta_{g, r}^{\Sigma}
$$

for the maximal pro- $\Sigma$ quotient of the kernel $N_{g, r}$ of the surjection $\pi_{1}\left(\mathcal{C}_{g, r} \backslash \bigcup_{i=1}^{r} \operatorname{Im}\left(s_{i}^{\mathcal{M}}\right)\right) \rightarrow \pi_{1}\left(\mathcal{M}_{g, r}\right)$ (see Remark 1.3.1 below).

(ii) We write

$$
\rho_{g, r}^{\Sigma}\left(\text { resp., } \rho_{g,[r]}^{\Sigma}\right)
$$

for the natural homomorphism determined by the above commutative diagram

$$
\begin{gathered}
\pi_{1}\left(\mathcal{M}_{g, r}\right) \longrightarrow \operatorname{Out}\left(\Delta_{g, r}^{\Sigma}\right) \\
\left(\operatorname{resp} ., \pi_{1}\left(\mathcal{M}_{g,[r]}\right) \longrightarrow \operatorname{Out}\left(\Delta_{g, r}^{\Sigma}\right)\right) .
\end{gathered}
$$

Moreover, we write

$$
\rho_{g, r}^{\Sigma \text {-geom }}\left(\text { resp., } \rho_{g,[r]}^{\Sigma \text {-geom }}\right)
$$

for the homomorphism

$$
\begin{gathered}
\pi_{1}\left(\mathcal{M}_{g, r} \otimes_{k} \bar{k}\right) \longrightarrow \operatorname{Out}\left(\Delta_{g, r}^{\Sigma}\right) \\
\left(\operatorname{resp} ., \pi_{1}\left(\mathcal{M}_{g,[r]} \otimes_{k} \bar{k}\right) \longrightarrow \operatorname{Out}\left(\Delta_{g, r}^{\Sigma}\right)\right)
\end{gathered}
$$


obtained as the restriction of $\rho_{g, r}^{\Sigma}$ (resp., $\left.\rho_{g,[r]}^{\Sigma}\right)$ to $\pi_{1}\left(\mathcal{M}_{g, r} \otimes_{k} \bar{k}\right) \subseteq$ $\pi_{1}\left(\mathcal{M}_{g, r}\right)$ (resp., $\left.\pi_{1}\left(\mathcal{M}_{g,[r]} \otimes_{k} \bar{k}\right) \subseteq \pi_{1}\left(\mathcal{M}_{g,[r]}\right)\right)$.

(iii) Let $S$ be a scheme that is connected and of finite type over $k$, and let $X=(C, D \subseteq C)$ be a hyperbolic curve of type $(g, r)$ over $S$. Then the classifying morphism $S \rightarrow \mathcal{M}_{g,[r]}$ of $X$ determines - up to $\pi_{1}\left(\mathcal{M}_{g,[r]} \otimes_{k}\right.$ $\bar{k}$ )-inner automorphism - a section $s_{X / S}$ of the natural exact sequence

$$
1 \longrightarrow \pi_{1}\left(\mathcal{M}_{g,[r]} \otimes_{k} \bar{k}\right) \longrightarrow \pi_{1}\left(\mathcal{M}_{g,[r]}\right) \times_{G_{k}} \pi_{1}(S) \longrightarrow \pi_{1}(S) \longrightarrow 1
$$

Thus, by considering the composite of $s_{X / S}$ and $\rho_{g,[r]}^{\Sigma}$, we obtain a homomorphism

$$
\rho_{X / S}^{\Sigma}: \pi_{1}(S) \longrightarrow \operatorname{Out}\left(\Delta_{g, r}^{\Sigma}\right)
$$

which is determined up to $\operatorname{Im}\left(\rho_{g,[r]}^{\Sigma \text {-geom }}\right)$-inner automorphism.

REMARK 1.3.1. It follows immediately from [11, Lemma 2.1], for example, that $\Delta_{g, r}^{\Sigma}$ is naturally isomorphic to the maximal pro- $\Sigma$ quotient of the fundamental group of the geometric fiber of the universal curve $\mathcal{C}_{g, r} \backslash$ $\bigcup_{i=1}^{r} \operatorname{Im}\left(s_{i}^{\mathcal{M}}\right) \rightarrow \mathcal{M}_{g, r}$ at a geometric point of $\mathcal{M}_{g, r}$. In particular, it follows immediately from [17, Corollary 1.3.4], for example, that $\Delta_{g, r}^{\Sigma}$ is slim (see the discussion on topological groups in Section 0); moreover, there exists a natural bijection between the following two sets:

- the set of cusps of the geometric fiber of the universal curve $\mathcal{C}_{g, r} \backslash$ $\bigcup_{i=1}^{r} \operatorname{Im}\left(s_{i}^{\mathcal{M}}\right) \rightarrow \mathcal{M}_{g, r}$ at a geometric point of $\mathcal{M}_{g, r}$;

- the set of conjugacy classes of cuspidal inertia subgroups of $\Delta_{g, r}^{\Sigma}$ associated to cusps of the geometric fiber of the universal curve $\mathcal{C}_{g, r} \backslash$ $\bigcup_{i=1}^{r} \operatorname{Im}\left(s_{i}^{\mathcal{M}}\right) \rightarrow \mathcal{M}_{g, r}$ at a geometric point of $\mathcal{M}_{g, r}$.

LEMMA 1.4 (kernels of the universal outer monodromy representations). We have the following.

(i) The action of $\pi_{1}\left(\mathcal{M}_{g,[r]}\right)$ on the set of conjugacy classes of cuspidal inertia subgroups of $\Delta_{g, r}^{\Sigma}$ induced by $\rho_{g,[r]}^{\Sigma}$ factors through the quotient $\pi_{1}\left(\mathcal{M}_{g,[r]}\right) \rightarrow \pi_{1}\left(\mathcal{M}_{g,[r]}\right) / \pi_{1}\left(\mathcal{M}_{g, r}\right) \simeq \mathfrak{S}_{r}$, and the resulting action of $\mathfrak{S}_{r}$ on the set of conjugacy classes of cuspidal inertia subgroups of $\Delta_{g, r}^{\Sigma}$ is faithful.

(ii) The kernel of $\rho_{g,[r]}^{\Sigma}$ is contained in $\pi_{1}\left(\mathcal{M}_{g, r}\right)$ and coincides with the kernel of $\rho_{g, r}^{\Sigma}$. 
Proof. Assertion (i) follows immediately from the various definitions involved, together with Remark 1.3.1. Assertion (ii) follows immediately from assertion (i), together with Remark 1.3.1.

Definition 1.5. Let $S$ be a scheme, and let $X=(C, D \subseteq C)$ be a hyperbolic curve of type $(g, r)$ over $S$. We have the following.

(i) We say that the hyperbolic curve $X$ is split if the finite étale covering obtained as the composite $D \hookrightarrow C \rightarrow S$ (see Definition 1.1(ii)) is trivial, that is, $D$ is isomorphic to the disjoint union of $r$ copies of $S$ over $S$.

(ii) Let $X_{0}=\left(C_{0}, D_{0} \subseteq C_{0}\right)$ be a hyperbolic curve over $S$. Then we say that $X_{0}$ is a hyperbolic partial compactification of $X$ if there exists an open immersion $C \backslash D \hookrightarrow C_{0} \backslash D_{0}$ over $S$.

(iii) Suppose that $g \geq 2$. Then it is immediate that the pair $(C, \emptyset \subseteq C)$ is a hyperbolic partial compactification of the hyperbolic curve $X$. We write $X^{\mathrm{cpt}}=(C, D \subseteq C)^{\mathrm{cpt}} \stackrel{\text { def }}{=}(C, \emptyset \subseteq C)$, and we refer to this as the compactification of $X$.

REMARK 1.5.1. Let $S$ be a scheme that is connected and of finite type over $k$, and let $X$ be a hyperbolic curve of type $(g, r)$ over $S$. We have the following.

(i) It follows immediately from Lemma 1.4(i) that the hyperbolic curve $X$ is split if and only if the image $\operatorname{Im}\left(\rho_{X / S}^{\Sigma}\right)$ is contained in the image $\operatorname{Im}\left(\rho_{g, r}^{\Sigma}\right)$

(ii) Let $X_{0}$ be a hyperbolic partial compactification of $X$. Then it follows immediately from the various definitions involved that the homomorphism $\rho_{X_{0} / S}^{\Sigma}$ factors through the homomorphism $\rho_{X / S}^{\Sigma}$; thus, we obtain natural surjections

$$
\pi_{1}(S) \rightarrow \operatorname{Im}\left(\rho_{X / S}^{\Sigma}\right) \rightarrow \operatorname{Im}\left(\rho_{X_{0} / S}^{\Sigma}\right)
$$

In particular, if $g \geq 2$, then we obtain natural surjections

$$
\pi_{1}(S) \rightarrow \operatorname{Im}\left(\rho_{X / S}^{\Sigma}\right) \rightarrow \operatorname{Im}\left(\rho_{X^{\mathrm{cpt}} / S}^{\Sigma}\right)
$$

LEMMA 1.6 (universal pro-l outer monodromy representations). Suppose that $\Sigma$ is of cardinality 1. Then the following hold. 
(i) The natural surjection $\pi_{1}\left(\mathcal{M}_{g, r}\right) \rightarrow G_{k}=\pi_{1}\left(\mathcal{M}_{0,3}\right)$ induces a surjection $\operatorname{Ker}\left(\rho_{g, r}^{\Sigma}\right) \rightarrow \operatorname{Ker}\left(\rho_{0,3}^{\Sigma}\right)$. In particular, we obtain a commutative diagram

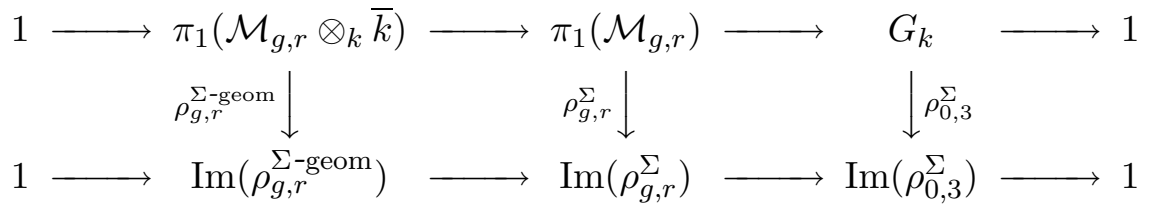

where the horizontal sequences are exact.

(ii) The natural surjection $\pi_{1}\left(\mathcal{M}_{g,[r]}\right) \rightarrow G_{k}=\pi_{1}\left(\mathcal{M}_{0,3}\right)$ induces a surjection $\operatorname{Ker}\left(\rho_{g,[r]}^{\Sigma}\right) \rightarrow \operatorname{Ker}\left(\rho_{0,3}^{\Sigma}\right)$. In particular, we obtain a commutative diagram

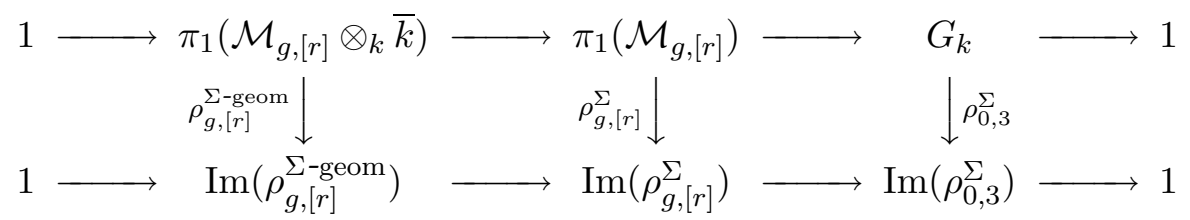

where the horizontal sequences are exact.

(iii) The commutative diagram

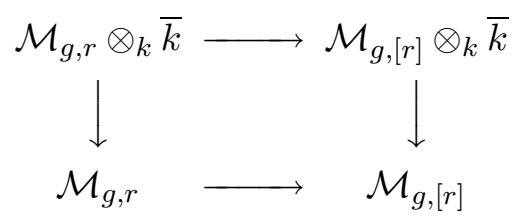

induces a commutative diagram

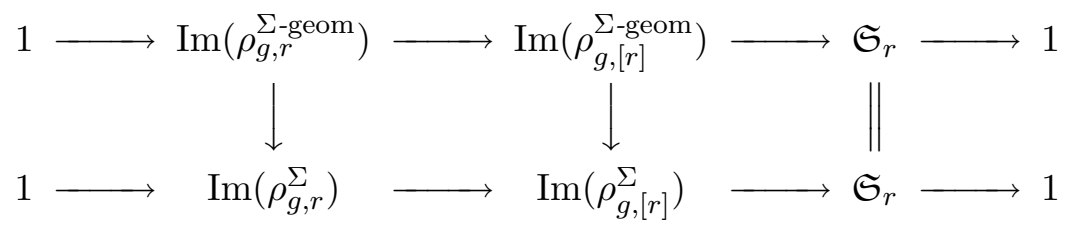

where the horizontal sequences are exact and where the vertical arrows are injective.

Proof. Assertion (i) is a consequence of a result concerning Oda's problem: If $r \neq 0$, then the desired surjectivity was proved in [9, Corollary 4.2.2]; on the other hand, if $r=0$, then the desired surjectivity follows from [9, Theorem 3B], together with [8], Theorem C, or a result obtained in [24].

Assertion (ii) follows immediately from assertion (i), together with Lemma 1.4(ii). Assertion (iii) follows immediately from Lemma 1.4(ii). 
For the remainder of Section 1, we consider the almost slimness (see the discussion on topological groups in Section 0) of the images of outer monodromy representations. Note that we do not use almost slimness later in this paper; only the slimness of the image of the homomorphism $\rho_{0, r}^{\{l\}}$ (see Lemma 4.3(ii) below) appears in the arguments of the proofs of the main results.

Proposition 1.7 (almost slimness of the images of outer monodromy representations). Let $H \subseteq \operatorname{Im}\left(\rho_{g,[r]}^{\Sigma}\right)$ be a closed subgroup of the image $\operatorname{Im}\left(\rho_{g,[r]}^{\Sigma}\right)$. Then the following hold.

(i) If $\Sigma$ consists of exactly one prime number $l$, then $H$ is almost pro-l (see the discussion on topological groups in Section 0).

(ii) Suppose that $k$ is a generalized sub-l-adic field (see the discussion on numbers in Section 0) for some $l \in \Sigma$, and suppose that there exists a hyperbolic curve $X$ of type $(g, r)$ over a finite extension $k^{\prime} \subseteq \bar{k}$ of $k$ such that $H$ contains the image $\operatorname{Im}\left(\rho_{X / k^{\prime}}^{\Sigma}\right)$. Then $H$ is almost slim (see the discussion on topological groups in Section 0). In particular, the images $\operatorname{Im}\left(\rho_{g, r}^{\Sigma}\right), \operatorname{Im}\left(\rho_{g,[r]}^{\Sigma}\right)$, and $\operatorname{Im}\left(\rho_{X / k^{\prime}}^{\Sigma}\right)$-where $X$ is a hyperbolic curve of type $(g, r)$ over a finite extension $k^{\prime} \subseteq \bar{k}$ of $k$-are almost slim.

Proof. First, we consider assertion (i). It follows from [1, Corollary 7], together with the fact that $\Delta_{g, r}^{\Sigma}$ is topologically finitely generated (see Remark 1.3.1) and pro-l, that the image $\operatorname{Im}\left(\rho_{g,[r]}^{\Sigma_{n}}\right)$ is almost pro- $l$. Thus, $H$ is almost pro- $l$, as desired. This completes the proof of assertion (i).

Next, we consider assertion (ii). Suppose that there exists a hyperbolic curve $X$ of type $(g, r)$ over a finite extension $k^{\prime} \subseteq \bar{k}$ of $k$ such that $H$ contains the image $\operatorname{Im}\left(\rho_{X / k^{\prime}}^{\Sigma}\right)$. Then, since $\Delta_{g, r}^{\Sigma}$ is center-free (see Remark 1.3.1), it follows from [14, Theorem 4.12] and [17, Corollary 1.5.7] that there exists a natural bijection

$$
\operatorname{Aut}_{\bar{k}}\left(X \otimes_{k^{\prime}} \bar{k}\right) \stackrel{\sim}{\longrightarrow} Z_{\text {Out }\left(\Delta_{g, r}^{\Sigma}\right)}^{\text {loc }}\left(\operatorname{Im}\left(\rho_{X / k^{\prime}}^{\Sigma}\right)\right)
$$

(see the discussion on topological groups in Section 0); in particular, $Z_{\text {Out }\left(\Delta_{g, r}^{\Sigma}\right)}^{\text {loc }}\left(\operatorname{Im}\left(\rho_{X / k^{\prime}}^{\Sigma}\right)\right)$ is finite. On the other hand, since $\operatorname{Im}\left(\rho_{X / k^{\prime}}^{\Sigma}\right) \subseteq H$, it follows that $Z_{\operatorname{Out}\left(\Delta_{g, r}^{\Sigma}\right)}^{\text {loc }}(H) \subseteq Z_{\operatorname{Out}\left(\Delta_{g, r}^{\Sigma}\right)}^{\operatorname{loc}}\left(\operatorname{Im}\left(\rho_{X / k^{\prime}}^{\Sigma}\right)\right.$ ) (see the discussion on topological groups in Section 0) is finite. Therefore, it follows from Lemma 1.8 below that $H$ is almost slim. This completes the proof of assertion (ii). 
LEMmA 1.8 (almost slimness and the finiteness of local center). Let $G$ be a profinite group. Then the following conditions are equivalent:

(i) $G$ is almost slim (see the discussion on topological groups in Section 0);

(ii) the local center $Z^{\mathrm{loc}}(G)$ (see the discussion on topological groups in Section 0) is finite.

Proof. First, to prove the implication

$$
\text { (i) } \Longrightarrow(\text { ii), }
$$

suppose that condition (i) is satisfied, that is, that there exists an open subgroup $H \subseteq G$ of $G$ that is slim. By replacing $H$ with a suitable open subgroup of $H$, we may assume without loss of generality that $H$ is normal in $G$. Now, since $H$ is slim, it follows that $Z^{\text {loc }}(G) \cap H=Z^{\text {loc }}(H)=\{1\}$. Thus, the composite $Z^{\mathrm{loc}}(G) \hookrightarrow G \rightarrow G / H$ is injective; in particular, $Z^{\text {loc }}(G)$ is finite. This completes the proof of the above implication. Finally, to prove the implication

$$
\text { (ii) } \Longrightarrow(\mathrm{i})
$$

suppose that condition (ii) is satisfied. Since $Z^{\text {loc }}(G) \subseteq G$ is finite, there exists an open subgroup $H \subseteq G$ of $G$ such that $Z^{\text {loc }}(G) \cap H=\{1\}$. On the other hand, since $Z^{\mathrm{loc}}(H)=Z^{\mathrm{loc}}(G) \cap H$, it follows that $Z^{\mathrm{loc}}(H)=\{1\}$, that is, $H$ is slim. This completes the proof of the above implication.

\section{§2. Monodromically full points and curves}

In this section, we define the notion of a $\Sigma$-monodromically full hyperbolic curve (see Definition 2.2 below), as well as the related notion of a $\Sigma$-monodromically full point (see Definition 2.1 below). Here, let $(g, r)$ be a pair of nonnegative integers such that $2 g-2+r>0$, and let $\Sigma$ be a nonempty set of prime numbers.

First, we define the notions of a $\Sigma$-monodromically full, strictly $\Sigma$-monodromically full, and quasi- $\Sigma$-monodromically full point.

Definition 2.1. Let $S$ be a scheme that is connected and of finite type over $k$, let $X=(C, D \subseteq C)$ be a hyperbolic curve of type $(g, r)$ over $S$, and let $s \in S$ be a closed point of $S$. Write $X_{s}$ for the hyperbolic curve over the residue field $k(s)$ of $S$ at $s$ obtained as the fiber of $X \rightarrow S$ at $s \in S$, that is, $X_{s}=\left(C \times{ }_{S} \operatorname{Spec} k(s), D \times{ }_{S} \operatorname{Spec} k(s)\right)$. We have the following. 
(i) We say that $s \in S$ is a $\Sigma$-monodromically full point with respect to $X / S$ if, for any $l \in \Sigma$, the closed subgroup $\operatorname{Im}\left(\rho_{X_{s} / k(s)}^{\{l\}}\right)$ of $\operatorname{Im}\left(\rho_{X / S}^{\{l l\}}\right)$ here, $\operatorname{Im}\left(\rho_{X_{s} / k(s)}^{\{l\}}\right)$ and $\operatorname{Im}\left(\rho_{X / S}^{\{l\}}\right)$ are determined up to $\operatorname{Im}\left(\rho_{g,[r]}^{\{l\} \text {-geom }}\right)$ conjugation-contains $\operatorname{Im}\left(\rho_{X / S}^{\{l\}}\right) \cap \operatorname{Im}\left(\rho_{g, r}^{\{l\}}\right)$.

(ii) We say that $s \in S$ is a strictly $\Sigma$-monodromically full point with respect to $X / S$ if, for any $l \in \Sigma$, the closed subgroup $\operatorname{Im}\left(\rho_{X_{s} / k(s)}^{\{l\}}\right)$ of $\operatorname{Im}\left(\rho_{X / S}^{\{l\}}\right)$ here, $\operatorname{Im}\left(\rho_{X_{s} / k(s)}^{\{l\}}\right)$ and $\operatorname{Im}\left(\rho_{X / S}^{\{l\}}\right)$ are determined up to $\operatorname{Im}\left(\rho_{g,[r]}^{\{l\} \text {-geom }}\right)$ conjugation - coincides with $\operatorname{Im}\left(\rho_{X / S}^{\{l\}}\right)$.

(iii) We say that $s \in S$ is a quasi- $\Sigma$-monodromically full point with respect to $X / S$ if, for any $l \in \Sigma$, the closed subgroup $\operatorname{Im}\left(\rho_{X_{s} / k(s)}^{\{l\}}\right)$ of $\operatorname{Im}\left(\rho_{X / S}^{\{l\}}\right)$ here, $\operatorname{Im}\left(\rho_{X_{s} / k(s)}^{\{l l\}}\right)$ and $\operatorname{Im}\left(\rho_{X / S}^{\{l\}}\right)$ are determined up to $\operatorname{Im}\left(\rho_{g,[r]}^{\{l\} \text {-geom }}\right)$ conjugation - is an open subgroup of $\operatorname{Im}\left(\rho_{X / S}^{\{l\}}\right)$.

If $l$ is a prime number, then, for simplicity, we write $l$-monodromically full (resp., strictly $l$-monodromically full, quasi-l-monodromically full) instead of $\{l\}$-monodromically full (resp., strictly $\{l\}$-monodromically full, quasi$\{l\}$-monodromically full).

REMARK 2.1.1. Let $S$ be a scheme that is connected and of finite type over $k$, let $X$ be a hyperbolic curve over $S$, and let $s \in S$ be a closed point of $S$. Consider the following conditions:

(i) $s \in S$ is strictly $\Sigma$-monodromically full with respect to $X / S$;

(ii) $s \in S$ is $\Sigma$-monodromically full with respect to $X / S$;

(iii) $s \in S$ is quasi- $\Sigma$-monodromically full with respect to $X / S$.

Then, as the terminologies suggest, it follows immediately from the various definitions involved that the implications

$$
\text { (i) } \Longrightarrow(\mathrm{ii}) \Longrightarrow(\mathrm{iii})
$$

hold.

Next, we define the notions of a $\Sigma$-monodromically full, strictly $\Sigma$-monodromically full, and quasi- $\Sigma$-monodromically full hyperbolic curve. Roughly speaking, a $\Sigma$-monodromically full (resp., strictly $\Sigma$-monodromically full, quasi- $\Sigma$-monodromically full) hyperbolic curve is one corresponding to a $\Sigma$ monodromically full (resp., strictly $\Sigma$-monodromically full, quasi- $\Sigma$-monodromically full) point of the moduli stack with respect to the universal curve. 
Definition 2.2. Let $X$ be a hyperbolic curve of type $(g, r)$ over $k$. We have the following.

(i) We say that $X$ is $\Sigma$-monodromically full if, for any $l \in \Sigma$, the closed subgroup $\operatorname{Im}\left(\rho_{X / k}^{\{l\}}\right)$-which is determined up to $\operatorname{Im}\left(\rho_{g,[r]}^{\{l\} \text {-geom }}\right)$-conjugation-of $\operatorname{Im}\left(\rho_{g,[r]}^{\{l\}}\right)$ contains $\operatorname{Im}\left(\rho_{g, r}^{\{l\}}\right)$.

(ii) We say that $X$ is strictly $\Sigma$-monodromically full if, for any $l \in \Sigma$, the closed subgroup $\operatorname{Im}\left(\rho_{X / k}^{\{l\}}\right)$-which is determined up to $\operatorname{Im}\left(\rho_{g,[r]}^{\{l\} \text {-geom }}\right)$ conjugation - of $\operatorname{Im}\left(\rho_{g,[r]}^{\{l\}}\right)$ contains $\operatorname{Im}\left(\rho_{g,[r]}^{\{l\} \text {-geom }}\right)$ or, equivalently, the closed subgroup $\operatorname{Im}\left(\rho_{X / k}^{\{l\}}\right)$ of $\operatorname{Im}\left(\rho_{g,[r]}^{\{l\}}\right)$ coincides with $\operatorname{Im}\left(\rho_{g,[r]}^{\{l l\}}\right)$.

(iii) We say that $X$ is quasi- $\Sigma$-monodromically full if, for any $l \in \Sigma$, the closed subgroup $\operatorname{Im}\left(\rho_{X / k}^{\{l\}}\right)$-which is determined up to $\operatorname{Im}\left(\rho_{g,[r]}^{\{l\} \text {-geom }}\right)$ conjugation - of $\operatorname{Im}\left(\rho_{g,[r]}^{\{l\}}\right)$ is an open subgroup of $\operatorname{Im}\left(\rho_{g,[r]}^{\{l\}}\right)$.

If $l$ is a prime number, then, for simplicity, we write $l$-monodromically full (resp., strictly l-monodromically full, quasi-l-monodromically full) instead of $\{l\}$-monodromically full (resp., strictly $\{l\}$-monodromically full, quasi- $\{l\}$ monodromically full).

Remark 2.2.1. Let $X$ be a hyperbolic curve over $k$. Consider the following conditions:

(i) $X$ is strictly $\Sigma$-monodromically full;

(ii) $X$ is $\Sigma$-monodromically full;

(iii) $X$ is quasi- $\Sigma$-monodromically full.

Then, as the terminologies suggest, it follows immediately from the various definitions involved that the implications

$$
\text { (i) } \Longrightarrow(\text { ii }) \Longrightarrow(\text { iii) }
$$

hold.

Remark 2.2.2. Let $X$ be a hyperbolic curve over $k$, and let $\Sigma_{1}, \Sigma_{2}$ be nonempty sets of prime numbers. Suppose that $\Sigma_{2} \subseteq \Sigma_{1}$. Consider the following conditions:

(i) $X$ is $\Sigma_{1}$-monodromically full (resp., strictly $\Sigma_{1}$-monodromically full, quasi- $\Sigma_{1}$-monodromically full),

(ii) $X$ is $\Sigma_{2}$-monodromically full (resp., strictly $\Sigma_{2}$-monodromically full, quasi- $\Sigma_{2}$-monodromically full). 
Then it follows immediately from the various definitions involved that the implication

$$
\text { (i) } \Longrightarrow(\mathrm{ii})
$$

holds.

REMARK 2.2.3. Let $X$ be a hyperbolic curve of type $(g, r)$ over $k$. Suppose that $r \leq 1$. Consider the following conditions:

(i) $X$ is $\Sigma$-monodromically full,

(ii) $X$ is strictly $\Sigma$-monodromically full.

Then it follows immediately from the various definitions involved that the equivalence

$$
\text { (i) } \Longleftrightarrow \text { (ii) }
$$

holds.

Remark 2.2.4. Let $X$ be a hyperbolic curve of type $(g, r)$ over $k$. Suppose that $r \geq 2$. Consider the following conditions:

(i) $X$ is strictly $\Sigma$-monodromically full,

(ii) $X$ is not split (see Definition 1.5(i)).

Then it follows immediately from Remark 1.5.1(i) that the implication

$$
\text { (i) } \Longrightarrow(\text { ii) }
$$

holds.

REMARK 2.2.5. Let $X_{1}$ be a hyperbolic curve over $k$, and let $X_{2}$ be a hyperbolic partial compactification of $X_{1}$ (see Definition 1.5(ii)). Consider the following conditions:

(i) $X_{1}$ is $\Sigma$-monodromically full (resp., strictly $\Sigma$-monodromically full, quasi- $\Sigma$-monodromically full),

(ii) $X_{2}$ is $\Sigma$-monodromically full (resp., strictly $\Sigma$-monodromically full, quasi- $\Sigma$-monodromically full).

Then it follows immediately from Remark 1.5.1(ii) that the implication

$$
\text { (i) } \Longrightarrow(\mathrm{ii})
$$

holds.

REMARK 2.2.6. Let $X$ be a hyperbolic curve over $k$, and let $k^{\prime} \subseteq \bar{k}$ be a finite extension of $k$. Consider the following conditions: 
(i) $X$ is a quasi- $\sum$-monodromically full hyperbolic curve over $k$,

(ii) $X \otimes_{k} k^{\prime}$ is a quasi- $\Sigma$-monodromically full hyperbolic curve over $k^{\prime}$.

Then it follows immediately from the various definitions involved that the equivalence

$$
\text { (i) } \Longleftrightarrow \text { (ii) }
$$

holds.

Remark 2.2.7. Let $X$ be a hyperbolic curve of type $(g, r)$ over $k$. Consider the following conditions:

(i) $X$ is split and $\Sigma$-monodromically full,

(ii) for any $l \in \Sigma$, the closed subgroup $\operatorname{Im}\left(\rho_{X / k}^{\{l\}}\right)$-which is determined up to $\operatorname{Im}\left(\rho_{g,[r]}^{\{l\} \text {-geom }}\right)$-conjugation-of $\operatorname{Im}\left(\rho_{g,[r]}^{\{l\}}\right)$ coincides with $\operatorname{Im}\left(\rho_{g, r}^{\{l\}}\right)$.

Then it follows immediately from Remark 1.5.1(i), together with the various definition involved, that the equivalence

$$
\text { (i) } \Longleftrightarrow \text { (ii) }
$$

holds.

REMARK 2.2.8. Let $S$ be a scheme that is connected and of finite type over $k$, let $X$ be a hyperbolic curve over $S$, and let $s \in S$ be a closed point of $S$. Write $k(s)$ for the residue field of $S$ at $s$, and write $X_{s}$ for the hyperbolic curve over $k(s)$ obtained as the fiber of $X \rightarrow S$ at $s \in S$ (see Definition 2.1). Consider the following conditions:

(i) $X_{s}$ is a $\Sigma$-monodromically full (resp., strictly $\Sigma$-monodromically full, quasi- $\Sigma$-monodromically full) hyperbolic curve over $k(s)$,

(ii) $s \in S$ is a $\Sigma$-monodromically full (resp., strictly $\Sigma$-monodromically full, quasi- $\Sigma$-monodromically full) point with respect to $X / S$.

Then it follows immediately from the various pertinent definitions that the implication

$$
\text { (i) } \Longrightarrow(\text { ii })
$$

holds.

The following result is essentially obtained in [11, Theorem 1.2]. Note that in [11], the following theorem in the case where $\Sigma$ is of cardinality 1 and where $k$ is a number field was proved. However, by an argument similar to that used in the proof of [11, Theorem 1.2], one may prove the following. 
TheOREM 2.3 (existence of many monodromically full points). Let $k$ be a finitely generated field of characteristic zero (see the discussion on numbers in Section 0), let $S$ be a scheme that is connected, regular, of finite type, and separated over $k$, let $S^{\mathrm{cl}}$ be the set of the closed points of $S$, let $X$ be a hyperbolic curve over $S$ (see Definition 1.1(ii)), let $\Sigma$ be a nonempty finite set of prime numbers, and let $S^{\mathrm{MF}} \subseteq S^{\mathrm{cl}}$ be the subset of $S^{\mathrm{cl}}$ consisting of closed points of $S$ which are strictly $\Sigma$-monodromically full with respect to $X / S$ (see Definition 2.1(ii)). Then if we naturally regard $S^{\mathrm{cl}}$, hence also $S^{\mathrm{MF}}$, as a subset of $S(\mathbb{C})$, then $S^{\mathrm{MF}} \subseteq S(\mathbb{C})$ is dense with respect to the complex topology of $S(\mathbb{C})$. Moreover, if $S$ is rational (i.e., there exists an open subscheme of $S$ which is isomorphic to an open subscheme of $\mathbb{P}_{k}^{n}$ for some positive integer $n)$, then the complement $S(k) \backslash\left(S(k) \cap S^{\mathrm{MF}}\right)$ in $S(k)$ of $S(k) \cap S^{\mathrm{MF}}$ forms a thin set in $S(k)$ in the sense of Hilbert's irreducibility theorem.

Proof. This follows from the fact that a finitely generated field of characteristic zero is Hilbertian, together with an argument similar to that used in the proof of [11, Theorem 1.2], by replacing [11, Lemma 3.1], (resp., [11, Lemma 3.3]) with Lemma 2.4 (resp., Lemma 2.5) below.

Lemma 2.4 (existence of a certain open subgroup). Let $G$ be a profinite group, let $\Sigma$ be a nonempty finite set of prime numbers, and, for each $l \in \Sigma$, let $G \rightarrow Q_{l}$ be a quotient of $G$ which is topologically finitely generated and almost pro-l (see the discussion on topological groups in Section 0). Then there exists a normal open subgroup $N \subseteq G$ of $G$ satisfying the following condition: if $H$ is a profinite group and if $H \rightarrow G$ is a continuous homomorphism such that the composite $H \rightarrow G \rightarrow G / N$ is surjective, then the composite $H \rightarrow G \rightarrow Q_{l}$ is surjective for each $l \in \Sigma$.

Proof. If $\Sigma$ is of cardinality 1, then Lemma 2.4 follows from [11, Lemma 3.1]. In particular, for each $l \in \Sigma$, there exists a normal open subgroup $N_{l} \subseteq G$ satisfying the following condition: if $H$ is a profinite group and if $H \rightarrow G$ is a continuous homomorphism such that the composite $H \rightarrow G \rightarrow G / N_{l}$ is surjective, then the composite $H \rightarrow G \rightarrow Q_{l}$ is surjective. Now write $N \stackrel{\text { def }}{=} \bigcap_{l \in \Sigma} N_{l} \subseteq G$. Then it is immediate that this normal open subgroup $N$ of $G$ satisfies the condition in the statement of Lemma 2.4. This completes the proof of Lemma 2.4.

LEMMA 2.5 (finite generation of the images of outer monodromy representations). Let $k$ be a finitely generated field of characteristic zero (see the 
discussion on numbers in Section 0), let $S$ be a scheme that is connected and of finite type over $k$, let $X$ be a hyperbolic curve over $S$, and let $l$ be a prime number. Suppose that $S$ is regular and separated over $k$. Then the quotient $\operatorname{Im}\left(\rho_{X / S}^{\{l\}}\right)$ of $\pi_{1}(S)$ is topologically finitely generated.

Proof. To verify Lemma 2.5 , it is immediate that by replacing $k$ with a finite extension of $k$, we may assume without loss of generality that $S$ is geometrically connected over $k$ and that $S$ has a $k$-rational point $s \in S(k)$. Then we have an exact sequence

$$
1 \longrightarrow \pi_{1}\left(S \otimes_{k} \bar{k}\right) \longrightarrow \pi_{1}(S) \longrightarrow G_{k} \longrightarrow 1
$$

Since $\pi_{1}\left(S \otimes_{k} \bar{k}\right)$ is topologically finitely generated (see [7, exposé II, théorème 2.3.1]), to verify Lemma 2.5 it suffices to show that the image of the composite

$$
G_{k} \longrightarrow \pi_{1}(S) \stackrel{\rho_{X / S}^{\{l\}}}{\longrightarrow} \operatorname{Out}\left(\Delta_{g, r}^{\{l\}}\right)
$$

where the first arrow is the outer homomorphism (which is determined up to $\pi_{1}\left(S \otimes_{k} \bar{k}\right)$-inner automorphism) induced by $s \in S(k)$ and where $(g, r)$ is the type of the hyperbolic curve $X$ over $S$, is topologically finitely generated. In particular, since the above composite coincides with the pro- $l$ outer monodromy representation $\rho_{X_{s} / k}^{\{l\}}$ associated to the hyperbolic curve $X_{s}$ over $k$ obtained as the fiber of $X \rightarrow S$ at $s \in S(k)$, to verify Lemma 2.5-by replacing $X$ with $X_{s}$-we may assume without loss of generality that $S=\operatorname{Spec} k$.

Since $k$ is a finitely generated field of characteristic zero, there exist a finite extension $k^{\prime} \subseteq \bar{k}$ of $k$, a subfield $k_{0} \subseteq k^{\prime}$ of $k^{\prime}$, and a scheme $V_{0}$ over $k_{0}$ satisfying the following conditions:

(i) $k_{0}$ is a number field (see the discussion on numbers in Section 0);

(ii) $V_{0}$ is regular, separated, geometrically connected, and of finite type over $k_{0}$;

(iii) $V_{0}$ has a $k_{0}$-rational point $v \in V_{0}\left(k_{0}\right)$;

(iv) the function field of $V_{0}$ is isomorphic to $k^{\prime}$;

(v) the hyperbolic curve $X \otimes_{k} k^{\prime}$ over $k^{\prime}$ extends to a hyperbolic curve $X_{0}$ over $V_{0}$.

Now, since the natural outer homomorphism $\pi_{1}\left(\operatorname{Spec} k^{\prime}\right) \rightarrow \pi_{1}\left(V_{0}\right)$ (see (iv)) is surjective (see (ii)) and since the pro- $l$ outer monodromy representation $\rho_{X \otimes_{k} k^{\prime} / k^{\prime}}^{\{l\}}$ factors through $\rho_{X_{0} / V_{0}}^{\{l l\}}($ see $(\mathrm{v}))$, to verify Lemma 2.5 it suffices to 
show that the image $\operatorname{Im}\left(\rho_{X_{0} / V_{0}}^{\{l\}}\right)$ is topologically finitely generated. Moreover, by the existence of the exact sequence (see (ii))

$$
1 \longrightarrow \pi_{1}\left(V_{0} \otimes_{k_{0}} \bar{k}_{0}\right) \longrightarrow \pi_{1}\left(V_{0}\right) \longrightarrow \operatorname{Gal}\left(\bar{k}_{0} / k_{0}\right) \longrightarrow 1,
$$

where $\bar{k}_{0}$ is the algebraic closure of $k_{0}$ determined by $\bar{k}$, together with the fact that $\pi_{1}\left(V_{0} \otimes_{k_{0}} \bar{k}_{0}\right)$ is topologically finitely generated (see [7, exposé II, théorème 2.3.1]), to verify Lemma 2.5 it suffices to show that the image of the composite

$$
\operatorname{Gal}\left(\bar{k}_{0} / k_{0}\right) \longrightarrow \pi_{1}\left(V_{0}\right) \stackrel{\rho_{X_{0} / V_{0}}^{\{l\}}}{\longrightarrow} \operatorname{Out}\left(\Delta_{g, r}^{\{l\}}\right)
$$

where the first arrow is the outer homomorphism (which is determined up to $\pi_{1}\left(V_{0} \otimes_{k_{0}} \bar{k}_{0}\right)$-inner automorphism) induced by $v \in V_{0}\left(k_{0}\right)$ (see (iii)), is topologically finitely generated. On the other hand, since $k_{0}$ is a number field (see (i)), it follows from [11, Lemma 3.1] that the image of the above composite is topologically finitely generated, as desired. This completes the proof of Lemma 2.5.

By Theorem 2.3, we obtain the following result.

COROLlary 2.6 (existence of many monodromically full hyperbolic curves). Let $k$ be a finitely generated field of characteristic zero (see the discussion on numbers in Section 0), let $\bar{k}$ be an algebraic closure of $k$, let $(g, r)$ be a pair of nonnegative integers such that $2 g-2+r>0$, let $\mathcal{M}_{g,[r]}$ be the moduli stack of hyperbolic curves of type $(g, r)$ over $k$-schemes (see Definition 1.2(ii)), let $M_{g,[r]}$ be the coarse moduli space associated to $\mathcal{M}_{g,[r]}$, and let $\Sigma$ be a nonempty finite set of prime numbers. Fix an inclusion $\bar{k} \hookrightarrow \mathbb{C}$. Then the subset of $M_{g,[r]}(\mathbb{C})$ of $\mathbb{C}$-valued points $s \in M_{g,[r]}(\mathbb{C})$ satisfying the following condition $(*)^{\mathrm{MF}}$ is dense with respect to the complex topology of $M_{g,[r]}(\mathbb{C})$.

$(*)^{\mathrm{MF}}$ : There exist a subfield $k^{\prime} \subseteq \bar{k}(\subseteq \mathbb{C})$ containing $k$ and a morphism $s_{k^{\prime}}:$ Spec $k^{\prime} \rightarrow \mathcal{M}_{g,[r]}$ over $k$ such that the hyperbolic curve corresponding to $s_{k^{\prime}}$ is a $\Sigma$-monodromically full hyperbolic curve over $k^{\prime}$ (see Definition 2.2(i)) and, moreover, such that $s: \operatorname{Spec} \mathbb{C} \rightarrow M_{g,[r]}$ factors through the composite $\operatorname{Spec} k^{\prime} \stackrel{s_{k^{\prime}}}{\longrightarrow} \mathcal{M}_{g,[r]} \rightarrow M_{g,[r]}$.

\section{§3. Relationship between monodromic fullness and certain prop- erties of hyperbolic curves}

In this section, we consider the relationship between monodromic fullness and certain properties of hyperbolic curves (see Propositions 3.4, 3.6, and 
3.8 below). Note that the facts proved here are not used in the proofs of the main results; however, Proposition 3.4 below appears in some arguments in Sections 7 and 8 . In Section 3, let $(g, r)$ be a pair of nonnegative integers such that $2 g-2+r>0$.

Definition 3.1. We write

$$
G_{g, r} \stackrel{\text { def }}{=} \begin{cases}\{1\} & (\text { if } 2 g-2+r \geq 3) \\ \mathbb{Z} / 2 \mathbb{Z} & \text { (if }(g, r)=(1,1),(1,2), \text { or }(2,0)) \\ \mathbb{Z} / 2 \mathbb{Z} \times \mathbb{Z} / 2 \mathbb{Z} & (\text { if }(g, r)=(0,4)) \\ \mathfrak{S}_{3} & (\text { if }(g, r)=(0,3)) .\end{cases}
$$

It seems to the author that the following proposition is likely to be well known.

Proposition 3.2 (automorphisms of general hyperbolic curves). Suppose that $k$ is algebraically closed. Then the following hold.

(i) If $X=(C, D \subseteq C)$ is a hyperbolic curve of type $(g, r)$ over $k$, then $G_{g, r}$ is isomorphic to a subgroup of the group $\operatorname{Aut}_{k}(X)$ of automorphisms of $X$ over $k$.

(ii) There exists a hyperbolic curve $X=(C, D \subseteq C)$ of type $(g, r)$ over $k$ such that the group $\operatorname{Aut}_{k}(X)$ of automorphisms of $X$ over $k$ is isomorphic to $G_{g, r}$.

Proof. First, we verify assertion (i). If $2 g-2+r \geq 3$, then assertion (i) is immediate. If $(g, r)=(0,3)$ or $(0,4)$, then assertion (i) may be verified by the fact that $\operatorname{Aut}_{k}(C)$ (note that $C$ is isomorphic to $\mathbb{P}_{k}^{1}$ over $k$ ) is isomorphic to $\mathrm{PGL}_{2}(k)$, together with a straightforward calculation. Note that if $(g, r)=(0,4)$, that is, $X=(C, D \subseteq C)$ is isomorphic to $\left(\mathbb{P}_{k}^{1},\{0,1, \infty, x\} \subseteq\right.$ $\left.\mathbb{P}_{k}^{1}\right)$ for some $x \in k \backslash\{0,1\}$, then the following two automorphisms generate a subgroup of $\operatorname{Aut}_{k}(X)$ which is isomorphic to $G_{0,4}=\mathbb{Z} / 2 \mathbb{Z} \times \mathbb{Z} / 2 \mathbb{Z}$ :

$$
\begin{gathered}
C \simeq \mathbb{P}_{k}^{1} \stackrel{\sim}{\longrightarrow} \mathbb{P}_{k}^{1} \simeq C ; \\
t / s \mapsto s x / t \\
C \simeq \mathbb{P}_{k}^{1} \stackrel{\sim}{\longrightarrow} \quad \mathbb{P}_{k}^{1} \simeq C . \\
t / s \stackrel{\mapsto}{\longrightarrow}(t-s) /(t-s x)
\end{gathered}
$$

Next, suppose that $(g, r)=(1,1)$ or $(1,2)$. If $(g, r)=(1,1)$ (resp., $(1,2))$, then write $\{o\}$ (resp., $\{o, x\}) \subseteq C(k)$ for the marked divisor $D$ of the hyperbolic curve $X=(C, D \subseteq C)$. Then, since $g=1$, by regarding the marked 
$k$-rational point $o$ of $C$ as an origin, one may regard $C$ as an abelian group scheme over $k$ whose identity section is the section determined by the $k$-rational point $o$. Thus, we have an automorphism

$$
\begin{cases}C \ni t \mapsto-t \in C & (\text { if } r=1) \\ C \ni t \mapsto x-t \in C & (\text { if } r=2)\end{cases}
$$

over $k$ of order 2 that preserves $D=\{o\}$ (resp., $=\{o, x\}) \subseteq C$; in particular, $G_{g, r}=\mathbb{Z} / 2 \mathbb{Z}$ is isomorphic to a subgroup of $\operatorname{Aut}_{k}(X)$. Next, suppose that $(g, r)=(2,0)$. Then, since the proper curve $C$ is hyperelliptic, we have an automorphism of $C$ of order 2 ; in particular, $G_{2,0}=\mathbb{Z} / 2 \mathbb{Z}$ is isomorphic to a subgroup of $\operatorname{Aut}_{k}(X)=\operatorname{Aut}_{k}(C)$. This completes the proof of assertion (i).

Finally, we verify assertion (ii). If $2 g-2+r \geq 3$, then assertion (ii) follows immediately, for example, from [12, Theorem C]. If $(g, r)=(0,3)$ or $(0,4)$, then assertion (ii) may be verified by the fact that $\operatorname{Aut}_{k}(C)$ (note that $C$ is isomorphic to $\mathbb{P}_{k}^{1}$ over $k$ ) is isomorphic to $\mathrm{PGL}_{2}(k)$, together with a straightforward calculation. Next, suppose that $(g, r)=(1,1)$ or $(1,2)$. Then, since $g=1$, one may regard $C$ as an abelian group scheme over $k$. Moreover, it is well known that there exists a hyperbolic curve $X=(C, D \subseteq C)$ of type $(1,1)$ (resp., $(1,2)$ ) over $k$ such that $\operatorname{Aut}_{k}(C)$ is isomorphic to $C(k) \rtimes\{ \pm 1\}$, where the action of $\{ \pm 1\}$ on $C(k)$ is the natural action of $\{ \pm 1\}$ on an abelian group $C(k)$. Now assertion (ii), in the case where $(g, r)=(1,1)$ or $(1,2)$, follows from the fact that $\operatorname{Aut}_{k}(C)$ is isomorphic to $C(k) \rtimes\{ \pm 1\}$, together with a straightforward calculation. Next, suppose that $(g, r)=(2,0)$. Then the assertion follows, for example, from [21, Theorem 1]. This completes the proof of assertion (ii).

Definition 3.3. Let $X$ be a hyperbolic curve of type $(g, r)$ over $k$. Then we say that $X$ has no special symmetry if the group $\operatorname{Aut}_{\bar{k}}\left(X \otimes_{k} \bar{k}\right)$ of automorphisms of $X \otimes_{k} \bar{k}$ over $\bar{k}$ is isomorphic to $G_{g, r}$.

Proposition 3.4 (quasi-monodromic fullness and automorphisms of hyperbolic curves). Let $X$ be a hyperbolic curve of type $(g, r)$ over $k$. Suppose that $X$ is quasi- $\Sigma$-monodromically full for a nonempty set of prime numbers $\Sigma$ and that $k$ is a generalized sub-l-adic field (see the discussion on numbers in Section 0) for some $l \in \Sigma$. Then $X$ has no special symmetry (see Definition 3.3).

Proof. Let $X_{0}$ be a hyperbolic curve of type $(g, r)$ over a finite extension $k_{0} \subseteq \bar{k}$ of $k$ such that $\operatorname{Aut}_{\bar{k}}\left(X_{0} \otimes_{k_{0}} \bar{k}\right) \simeq G_{g, r}$ (see Lemma 3.2(ii)). Then, since 
$\Delta_{g, r}^{\Sigma}$ is center-free (see Remark 1.3.1), it follows from [14, Theorem 4.12] and $[17$, Corollary 1.5.7] that there exist natural bijections

$$
\begin{gathered}
\operatorname{Aut}_{\bar{k}}\left(X \otimes_{k} \bar{k}\right) \stackrel{\sim}{\longrightarrow} Z_{\mathrm{Out}\left(\Delta_{g, r}^{\Sigma}\right)}^{\operatorname{loc}}\left(\operatorname{Im}\left(\rho_{X / k}^{\Sigma}\right)\right), \\
G_{g, r} \stackrel{\sim}{\longrightarrow} \operatorname{Aut}_{\bar{k}}\left(X_{0} \otimes_{k} \bar{k}\right) \stackrel{\sim}{\longrightarrow} Z_{\mathrm{Out}\left(\Delta_{g, r}^{\Sigma}\right)}^{\operatorname{loc}}\left(\operatorname{Im}\left(\rho_{X_{0} / k_{0}}^{\Sigma}\right)\right) .
\end{gathered}
$$

On the other hand, since $X$ is quasi- $\Sigma$-monodromically full, it follows immediately from the definition of the term "quasi- $\Sigma$-monodromically full" that

$$
Z_{\operatorname{Out}\left(\Delta_{g, r}^{\Sigma}\right)}^{\operatorname{loc}}\left(\operatorname{Im}\left(\rho_{X / k}^{\Sigma}\right)\right)=Z_{\operatorname{Out}\left(\Delta_{g, r}^{\Sigma}\right)}^{\operatorname{loc}}\left(\operatorname{Im}\left(\rho_{g,[r]}^{\Sigma}\right)\right)
$$

(see the discussion on topological groups in Section 0). Thus, since $\operatorname{Im}\left(\rho_{X_{0} / k_{0}}^{\Sigma}\right) \subseteq \operatorname{Im}\left(\rho_{g,[r]}^{\Sigma}\right)$, we obtain

$$
\begin{aligned}
\operatorname{Aut}_{\bar{k}}\left(X \otimes_{k} \bar{k}\right) & \stackrel{\sim}{\longrightarrow} Z_{\mathrm{Out}\left(\Delta_{g, r}^{\Sigma}\right)}^{\operatorname{loc}}\left(\operatorname{Im}\left(\rho_{X / k}^{\Sigma}\right)\right)=Z_{\mathrm{Out}\left(\Delta_{g, r}^{\Sigma}\right)}^{\operatorname{loc}}\left(\operatorname{Im}\left(\rho_{g,[r]}^{\Sigma}\right)\right) \\
& \subseteq Z_{\mathrm{Out}\left(\Delta_{g, r}^{\Sigma}\right)}^{\operatorname{loc}}\left(\operatorname{Im}\left(\rho_{X_{0} / k_{0}}^{\Sigma}\right)\right) \simeq G_{g, r}
\end{aligned}
$$

(see the discussion on topological groups in Section 0). In particular, it follows immediately from Lemma 3.2(i) that $X$ has no special symmetry. This completes the proof of Proposition 3.4.

Definition 3.5. Let $X$ be a hyperbolic curve of type $(g, r)$ over $k$, and let $\Sigma$ be a nonempty set of prime numbers. Suppose that $g \neq 0$. Then we say that $X$ is of $\Sigma$-AIJ-type if the following condition is satisfied. For any prime number $l \in \Sigma$ and finite extension $k^{\prime} \subseteq \bar{k}$ of $k$ such that $X\left(k^{\prime}\right) \neq \emptyset$, the $l$-adic Tate module of the Jacobian variety of the compactification of the hyperbolic curve $X \otimes_{k} k^{\prime}$ over $k^{\prime}$ is irreducible as a $G_{k^{\prime}}$-module.

REMARK 3.5.1. It follows immediately from the definition of the term of AIJ-type that if a hyperbolic curve $X$ over $k$ is of $\Sigma$-AIJ-type for some nonempty set of prime numbers $\Sigma$, then the Jacobian variety of the compactification of the hyperbolic curve $X \otimes_{k} \bar{k}$ over $\bar{k}$ is simple.

Proposition 3.6 (quasi-monodromic fullness and the absolute irreducibility of Jacobian variety). Let $X$ be a hyperbolic curve of type $(g, r)$ over $k$, and let $\Sigma$ be a nonempty set of prime numbers. Suppose that $k$ is a finitely generated field of characteristic zero (see the discussion on numbers in Section 0), that $g \neq 0$, and that $X$ is quasi- $\Sigma$-monodromically full. Then $X$ is of $\Sigma$-AIJ-type (see Definition 3.5). In particular, the Jacobian variety of the compactification of the hyperbolic curve $X \otimes_{k} \bar{k}$ over $\bar{k}$ is simple (see Remark 3.5.1). 
Proof. To prove Proposition 3.6, it follows from the definition of the term of AIJ-type that we may assume without loss of generality that $\Sigma$ is of cardinality 1 , that is, $\Sigma=\{l\}$ for some prime number $l$. Write $H_{g, r}^{\Sigma}$ for the abelian quotient of $\Delta_{g, r}^{\Sigma}$ by the normal closed subgroup generated by the cuspidal inertia subgroups of $\Delta_{g, r}^{\Sigma}$ and the closure of the commutator subgroup of $\Delta_{g, r}^{\Sigma}$. (Thus, if $g \geq 2$, then $H_{g, r}^{\Sigma}$ is naturally isomorphic to $\left(\Delta_{g, 0}^{\Sigma}\right)^{\text {ab }}$.) It now follows from an argument similar to that used in Remark 1.5.1(ii) that the pro- $\Sigma$ outer representation $\rho_{g,[r]}^{\Sigma}: \pi_{1}\left(\mathcal{M}_{g,[r]}\right) \rightarrow \operatorname{Out}\left(\Delta_{g, r}^{\Sigma}\right)$ induces a pro- $\Sigma$ representation $\rho: \pi_{1}\left(\mathcal{M}_{g,[r]}\right) \rightarrow \operatorname{Aut}\left(H_{g, r}^{\Sigma}\right)$. Moreover, it is well known that my following summary holds (see also Remark 1.3.1).

Summary: Let $k^{\prime} \subseteq \bar{k}$ be a finite extension of $k$ such that $X\left(k^{\prime}\right) \neq \emptyset$. Then there exists an isomorphism of $H_{g, r}^{\Sigma}$ with the $l$-adic Tate module of the Jacobian variety of the compactification of $X \otimes_{k} k^{\prime}$ such that, under this isomorphism, the action of $G_{k^{\prime}}$ on $H_{g, r}^{\Sigma}$ determined by $\rho, s_{X / k}$ (see Definition 1.3(iii)) and the natural action of $G_{k^{\prime}}$ on the $l$-adic Tate module coincide.

Therefore, Proposition 3.6 follows from the definition of the term quasimonodromically full, together with the existence of a hyperbolic curve of $\Sigma$-AIJ-type over a number field (see, e.g., [4, proof of Proposition 4]; see also [4, Remark 5(iv),(v)]).

Definition 3.7. Let $X$ be a hyperbolic curve of type $(g, r)$ over $k$. Suppose that $g \neq 0$. Then we say that $X$ has a JCM-component if there exist a nontrivial simple abelian variety $A$ over $\bar{k}$ such that $\operatorname{End}_{\bar{k}}(A) \otimes_{\mathbb{Z}} \mathbb{Q}$ is isomorphic to a number field of degree $2 \operatorname{dim}(A)$ and a nontrivial morphism over $\bar{k}$ from $A$ to the Jacobian variety of the compactification of the hyperbolic curve $X \otimes_{k} \bar{k}$.

Remark 3.7.1. Let $X$ be a hyperbolic curve of type $(1,1)$ over $k$. Then it follows from the various definitions involved that $X$ has a JCM-component if and only if the elliptic curve determined by $X$ admits complex multiplication over $\bar{k}$; that is, the ring of endomorphisms of the elliptic curve determined by $X$ over $\bar{k}$ is isomorphic to an order of an imaginary quadratic field.

Proposition 3.8 (quasi-monodromic fullness and complex multiplication). Let $X$ be a hyperbolic curve of type $(g, r)$ over $k$. Suppose that $k$ is a finitely generated field of characteristic zero (see the discussion on numbers in Section 0), that $g \neq 0$, and that $X$ is quasi- $\Sigma$-monodromically full for a nonempty set of prime numbers $\Sigma$. Then $X$ does not have a JCM-component (see Definition 3.7). 
Proof. This follows immediately from Proposition 3.6, together with [23, Corollary 2 on p. 502 to Theorem 5].

\section{§4. Moduli stacks of hyperbolic curves of genus zero}

In this section, we consider the moduli stacks of hyperbolic curves of genus zero. Here, let $r \geq 3$ be an integer, and let $l$ be a prime number.

LEMMA 4.1 (moduli stacks of hyperbolic curves of genus zero). We have the following.

(i) The moduli stack $\mathcal{M}_{0, r}$ is isomorphic to the $(r-3)$-rd configuration space of $\mathbb{P}_{k}^{1} \backslash\{0,1, \infty\}$ over $k$, that is, the open subscheme of the fiber product over $k$ of $r-3$ copies of $\mathbb{P}_{k}^{1} \backslash\{0,1, \infty\}$ obtained as the complement of the various diagonal divisors.

(ii) The natural homomorphism $\mathfrak{S}_{r} \rightarrow \operatorname{Aut}_{k}\left(\mathcal{M}_{0, r}\right)$ determined by the $\mathfrak{S}_{r}$ covering $\mathcal{M}_{0, r} \rightarrow \mathcal{M}_{0,[r]}$ is surjective. In particular, any automorphism $\phi$ of $\mathcal{M}_{0, r}$ over $k$ is an automorphism over $\mathcal{M}_{0,[r]}$; that is, there exists a commutative diagram

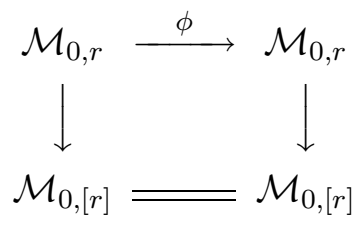

where the vertical arrows are natural morphisms and where the lower horizontal arrow is the identity automorphism of $\mathcal{M}_{0,[r]}$.

Proof. Assertions (i) and (ii) are well known. (Concerning assertion (ii), see also [17, Section 0] and the discussion following Theorem A.)

LEMMA 4.2 (universal geometric monodromy outer representations of genus zero). We have the following.

(i) The quotient $\pi_{1}\left(\mathcal{M}_{0, r} \otimes_{k} \bar{k}\right) \rightarrow \operatorname{Im}\left(\rho_{0, r}^{\{l\} \text {-geom }}\right)$ of $\pi_{1}\left(\mathcal{M}_{0, r} \otimes_{k} \bar{k}\right)$ coincides with the maximal pro-l quotient of $\pi_{1}\left(\mathcal{M}_{0, r} \otimes_{k} \bar{k}\right)$. In particular, there exists a natural homomorphism

$$
\operatorname{Aut}_{G_{k}}\left(\pi_{1}\left(\mathcal{M}_{0, r}\right)\right) \longrightarrow \operatorname{Aut}_{\operatorname{Im}\left(\rho_{0,3}^{\{l\}}\right)}\left(\operatorname{Im}\left(\rho_{0, r}^{\{l\}}\right)\right)
$$

(see Lemma 1.6(i)).

(ii) The abelianization of $\operatorname{Im}\left(\rho_{0, r}^{\{l\} \text {-geom }}\right)$ is a free $\mathbb{Z}_{l}$-module of rank $r(r-3) / 2$. 
Proof. Assertion (i) follows from [3, remark following the proof of Theorem 1], together with Lemma 4.1(i). Assertion (ii) follows immediately from [18, Corollary 2.5], together with Lemma 4.1(i). (Indeed, it follows from [18, Corollary 2.5], together with Lemma 4.1(i), that $\operatorname{rank}_{\mathbb{Z}_{l}}\left(\operatorname{Im}\left(\rho_{0, r}^{\{l\}-\text { geom }}\right)^{\mathrm{ab}}\right)=$ $\left.\sum_{i=3}^{r-1} \operatorname{rank}_{\mathbb{Z}_{l}}\left(\left(\Delta_{0, i}^{\{l\}}\right)^{\mathrm{ab}}\right)=\sum_{i=3}^{r-1}(i-1)=r(r-3) / 2.\right)$

LEMMA 4.3 (universal monodromy outer representations of genus zero). Suppose that $k$ is a finitely generated field of characteristic zero (see the discussion on numbers in Section 0). Then the following hold.

(i) The image $\operatorname{Im}\left(\rho_{0, r}^{\{l\} \text {-geom }}\right)$ is pro-l and slim.

(ii) The image $\operatorname{Im}\left(\rho_{0, r}^{\{l\}}\right)$ is slim. Moreover, if $k$ contains a primitive lth root of unity, then the image $\operatorname{Im}\left(\rho_{0, r}^{\{l\}}\right)$ is pro-l.

(iii) The composite of natural homomorphisms

$$
\begin{gathered}
\operatorname{Aut}_{k}\left(\mathcal{M}_{0, r}\right) \longrightarrow \operatorname{Aut}_{G_{k}}\left(\pi_{1}\left(\mathcal{M}_{0, r}\right)\right) / \operatorname{Inn}\left(\pi_{1}\left(\mathcal{M}_{0, r} \otimes_{k} \bar{k}\right)\right) \\
\longrightarrow \operatorname{Aut}_{\operatorname{Im}\left(\rho_{0,3}^{\{l\}}\right)}\left(\operatorname{Im}\left(\rho_{0, r}^{\{l\}}\right)\right) / \operatorname{Inn}\left(\operatorname{Im}\left(\rho_{0, r}^{\{l\}-\text { geom }}\right)\right)
\end{gathered}
$$

(see Lemma 4.2(i)) is bijective (see Remark 4.3.1).

(iv) The composite of natural maps

$$
\begin{gathered}
\mathcal{M}_{0, r}(k) \longrightarrow \operatorname{Hom}_{G_{k}}\left(G_{k}, \pi_{1}\left(\mathcal{M}_{0, r}\right)\right) / \operatorname{Inn}\left(\pi_{1}\left(\mathcal{M}_{0, r} \otimes_{k} \bar{k}\right)\right) \\
\longrightarrow \operatorname{Hom}_{\operatorname{Im}\left(\rho_{0,3}^{\{l\}}\right)}\left(G_{k}, \operatorname{Im}\left(\rho_{0, r}^{\{l\}}\right)\right) / \operatorname{Inn}\left(\operatorname{Im}\left(\rho_{0, r}^{\{l\} \text {-geom }}\right)\right)
\end{gathered}
$$

(see Lemma 4.2(i)) is injective.

(v) The composite of natural maps

$$
\begin{gathered}
\mathcal{M}_{0,[r]}(k) \longrightarrow \operatorname{Hom}_{G_{k}}\left(G_{k}, \pi_{1}\left(\mathcal{M}_{0,[r]}\right)\right) / \operatorname{Inn}\left(\pi_{1}\left(\mathcal{M}_{0,[r]} \otimes_{k} \bar{k}\right)\right) \\
\longrightarrow \operatorname{Hom}_{\operatorname{Im}\left(\rho_{0,3}^{\{l\}}\right)}\left(G_{k}, \operatorname{Im}\left(\rho_{0,[r]}^{\{l\}}\right)\right) / \operatorname{Inn}\left(\operatorname{Im}\left(\rho_{0,[r]}^{\{l\} \text {-geom }}\right)\right)
\end{gathered}
$$

(see Lemma 4.2(i)) is injective.

Proof. Assertion (i) follows from [16, Proposition 2.2(ii)], together with Lemmas 4.1(i) and 4.2(i).

Next, we verify assertion (ii). Since we have an exact sequence

$$
1 \longrightarrow \operatorname{Im}\left(\rho_{0, r}^{\{l\} \text {-geom }}\right) \longrightarrow \operatorname{Im}\left(\rho_{0, r}^{\{l\}}\right) \longrightarrow \operatorname{Im}\left(\rho_{0,3}^{\{l\}}\right) \longrightarrow 1
$$


(see Lemma 1.6(i)), it follows from assertion (i) that to verify the fact that $\operatorname{Im}\left(\rho_{0, r}^{\{l\}}\right)$ is slim (resp., pro- $l$ ), it suffices to show that $\operatorname{Im}\left(\rho_{0,3}^{\{l\}}\right)$ is $\operatorname{slim}$ (resp., pro- $l)$. Now we prove the fact that $\operatorname{Im}\left(\rho_{0,3}^{\{l\}}\right)$ is slim. It follows from an argument similar to that used in the proof of Proposition 1.7(ii), together with Lemma 4.1(i), that we obtain a natural bijection

$$
\operatorname{Aut}_{\bar{k}}\left(\mathbb{P}_{\bar{k}} \backslash\{0,1, \infty\}\right) \stackrel{\sim}{\longrightarrow} Z_{\operatorname{Out}\left(\Delta_{0,3}^{\{l\}}\right)}^{\operatorname{loc}}\left(\operatorname{Im}\left(\rho_{0,3}^{\{l\}}\right)\right) .
$$

Therefore, by comparing the natural actions of $\operatorname{Aut}_{\bar{k}}\left(\mathbb{P}_{\bar{k}} \backslash\{0,1, \infty\}\right)$ and $\operatorname{Im}\left(\rho_{0,3}^{\{l\}}\right)$ on the set of conjugacy classes of cuspidal inertia subgroups of $\Delta_{0,3}^{\{l\}}$ (see Remark 1.3.1), it follows that the intersection

$$
Z_{\operatorname{Out}\left(\Delta_{0,3}^{\{l\}}\right)}^{\operatorname{loc}}\left(\operatorname{Im}\left(\rho_{0,3}^{\{l\}}\right)\right) \cap \operatorname{Im}\left(\rho_{0,3}^{\{l\}}\right)
$$

is trivial. In particular, the local center $Z^{\operatorname{loc}}\left(\operatorname{Im}\left(\rho_{0,3}^{\{l\}}\right)\right)$ of $\operatorname{Im}\left(\rho_{0,3}^{\{l\}}\right)$ is trivial. This completes the proof of the fact that $\operatorname{Im}\left(\rho_{0,3}^{\{l\}}\right)$ is slim. On the other hand, it follows immediately from [2, Theorems A, B] that if $k$ contains a primitive $l$ th root of unity, then $\operatorname{Im}\left(\rho_{0,3}^{\{l\}}\right)$ is pro- $l$. This completes the proof of assertion (ii).

Next, we prove assertion (iii). By considering the action of $\operatorname{Aut}_{k}\left(\mathcal{M}_{0, r}\right)$ on the set of conjugacy classes of cuspidal inertia subgroups of $\operatorname{Im}\left(\rho_{0, r}^{\{l\} \text {-geom }}\right)$, the injectivity of the composite in question follows immediately from Lemmas 4.1(i),(ii) and 4.2(i), together with Remark 1.3.1. Now we verify the surjectivity of the composite in question by induction on $r$. If $r=3$, 4, then the surjectivity of the composite in question follows from [14, Theorem 4.12], together with Lemmas 4.1(i) and 4.2(i). Suppose that $r \geq 5$ and that the composite of natural homomorphisms

$$
\begin{gathered}
\operatorname{Aut}_{k}\left(\mathcal{M}_{0, r-1}\right) \longrightarrow \operatorname{Aut}_{G_{k}}\left(\pi_{1}\left(\mathcal{M}_{0, r-1}\right)\right) / \operatorname{Inn}\left(\pi_{1}\left(\mathcal{M}_{0, r-1} \otimes_{k} \bar{k}\right)\right) \\
\longrightarrow \operatorname{Aut}_{\operatorname{Im}\left(\rho_{0,3}^{\{l\}}\right)}\left(\operatorname{Im}\left(\rho_{0, r-1}^{\{l\}}\right)\right) / \operatorname{Inn}\left(\operatorname{Im}\left(\rho_{0, r-1}^{\{l\} \text {-geom }}\right)\right)
\end{gathered}
$$

is bijective. Let $\alpha$ be an automorphism of $\operatorname{Im}\left(\rho_{0, r}^{\{l\}}\right)$ over $\operatorname{Im}\left(\rho_{0,3}^{\{l\}}\right)$. Then it follows immediately from [17, Theorem 3.1.13] (note that one may easily verify that [17, Theorem 3.1.13] is valid for a finitely generated field of characteristic zero, even though in [17] this result for a number field is only stated) that-by compositing a suitable automorphism of $\operatorname{Im}\left(\rho_{0, r}^{\{l\}}\right)$ over $\operatorname{Im}\left(\rho_{0,3}^{\{l\}}\right)$ 
arising from an element of $\operatorname{Aut}_{k}\left(\mathcal{M}_{0, r}\right)$ - we may assume without loss of

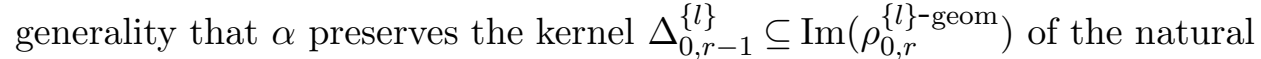
surjection $\operatorname{Im}\left(\rho_{0, r}^{\{l\}}\right) \rightarrow \operatorname{Im}\left(\rho_{0, r-1}^{\{l\}}\right)$ (see Lemmas 4.1(i) and 4.2(i)). Moreover, it follows immediately from the induction hypothesis that - again by compositing a suitable automorphism of $\operatorname{Im}\left(\rho_{0, r}^{\{l\}}\right)$ over $\operatorname{Im}\left(\rho_{0,3}^{\{l\}}\right)$ arising from an element of $\operatorname{Aut}_{k}\left(\mathcal{M}_{0, r}\right)$-we may assume without loss of generality that the automorphism of $\operatorname{Im}\left(\rho_{0, r-1}^{\{l\}}\right)$ induced by $\alpha$ is the identity automorphism of $\operatorname{Im}\left(\rho_{0, r-1}^{\{l\}}\right)$; that is, we obtain a commutative diagram

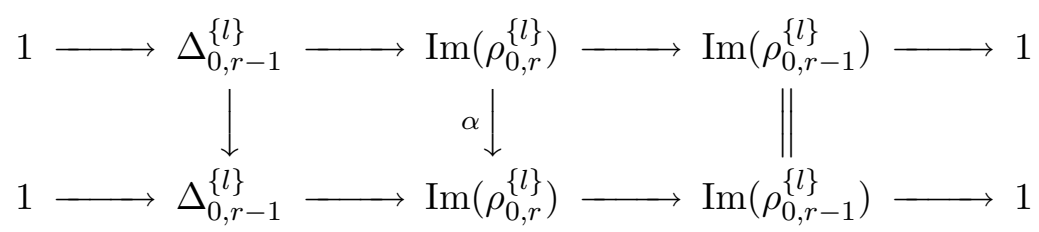

where the horizontal sequences are exact and where the right-hand vertical arrow is the identity automorphism. Therefore, it follows immediately from [14, Theorem 4.12], together with Lemma 4.4(ii), below, that $\alpha$ arises from an automorphism of $\mathcal{M}_{0, r}$ over $k$. This completes the proof of assertion (iii).

Assertion (iv) follows immediately from [13, Theorem C], together with Lemmas 4.1(i) and 4.2(i). Assertion (v) follows from [14, Remark] following Theorem 4.12 (see also [13, proof of Theorem C]).

REMARK 4.3.1. The bijectivity of the composite of natural homomorphisms

$$
\begin{gathered}
\operatorname{Aut}_{k}\left(\mathcal{M}_{0, r}\right) \longrightarrow \operatorname{Aut}_{G_{k}}\left(\pi_{1}\left(\mathcal{M}_{0, r}\right)\right) / \operatorname{Inn}\left(\pi_{1}\left(\mathcal{M}_{0, r} \otimes_{k} \bar{k}\right)\right) \\
\longrightarrow \operatorname{Aut}_{\operatorname{Im}\left(\rho_{0,3}^{\{l\}}\right)}\left(\operatorname{Im}\left(\rho_{0, r}^{\{l\}}\right)\right) / \operatorname{Inn}\left(\operatorname{Im}\left(\rho_{0, r}^{\{l\} \text {-geom }}\right)\right)
\end{gathered}
$$

in the case where $l$ is odd was proved in [17, Theorem A].

Lemma 4.4. Let $S$ be a connected normal scheme, and let $\eta_{S} \rightarrow S$ be the generic point of $S$. Then the following hold.

(i) Let $T \rightarrow S$ be a scheme that is finite over $S$. Then the natural morphism $\operatorname{Hom}_{S}(S, T) \rightarrow \operatorname{Hom}_{S}\left(\eta_{S}, T\right)$ is bijective.

(ii) Let $X_{1}, X_{2}$ be hyperbolic curves over $S$. Then the natural morphism

$$
\operatorname{Isom}_{S}\left(X_{1}, X_{2}\right) \longrightarrow \operatorname{Isom}_{\eta_{S}}\left(X_{1} \times_{S} \eta_{S}, X_{2} \times_{S} \eta_{S}\right)
$$

is bijective. 
Proof. First, we consider assertion (i). The injectivity of the morphism in question follows immediately from the fact that the natural morphism $\eta_{S} \rightarrow$ $S$ is scheme-theoretically dense. To verify the surjectivity of the morphism in question, let $\phi: \eta_{S} \rightarrow T$ be a morphism over $S$. Write $F \subseteq T$ for the scheme-theoretic image of $\phi$. Then it follows immediately from the various definitions involved that $F$ is integral and that the composite $F \hookrightarrow T \rightarrow S$ is birational and finite. Thus, since $S$ is normal, it follows from Zariski's main theorem (see [6, corollaire 4.4.9]) that the composite $F \hookrightarrow T \rightarrow S$ is an isomorphism. In particular, $\phi$ extends to a morphism $S \rightarrow T$ over $S$.

Finally, we consider assertion (ii). It follows from [5, Theorem 1.11], for example, that the functor

$$
T \rightsquigarrow \operatorname{Isom}_{T}\left(X_{1} \times_{S} T, X_{2} \times_{S} T\right)
$$

from the category of schemes over $S$ to the category of sets is represented by a scheme that is finite and unramified over $S$. Thus, assertion (ii) follows from assertion (i).

\section{§5. A Grothendieck conjecture-type lemma for certain images of the universal monodromy}

In this section, we prove a Grothendieck conjecture-type lemma for certain images of the universal monodromy (see Lemma 5.2 below). Here, let $r \geq 3$ be an integer, and let $l$ be a prime number. Moreover, suppose that $k$ is a finitely generated field of characteristic zero (see the discussion on numbers in Section 0). Let us fix an isomorphism $\operatorname{Im}\left(\rho_{0,[r]}^{\{l\}}\right) / \operatorname{Im}\left(\rho_{0, r}^{\{l\}}\right) \stackrel{\sim}{\rightarrow} \mathfrak{S}_{r}$ (see Lemma 1.6(iii)).

For $i=1,2$, let

$$
H_{i} \subseteq \operatorname{Im}\left(\rho_{0,[r]}^{\{l\}}\right)
$$

be an open subgroup of $\operatorname{Im}\left(\rho_{0,[r]}^{\{l\}}\right)$ that contains the normal open subgroup $\operatorname{Im}\left(\rho_{0, r}^{\{l\}}\right) \subseteq \operatorname{Im}\left(\rho_{0,[r]}^{\{l\}}\right)$, let

$$
\left(H_{i} \rightarrow\right) Q_{i}\left(\subseteq \mathfrak{S}_{r}\right)
$$

be the image of the composite $H_{i} \hookrightarrow \operatorname{Im}\left(\rho_{0,[r]}^{\{l\}}\right) \rightarrow \operatorname{Im}\left(\rho_{0,[r]}^{\{l\}}\right) / \operatorname{Im}\left(\rho_{0, r}^{\{l\}}\right) \simeq \mathfrak{S}_{r}$, and let

$$
H_{i}^{\text {geom }} \subseteq H_{i}
$$

be the kernel of the composite $H_{i} \hookrightarrow \operatorname{Im}\left(\rho_{0,[r]}^{\{l\}}\right) \rightarrow \operatorname{Im}\left(\rho_{0,3}^{\{l\}}\right)$, that is, $H_{i}^{\text {geom def }}=$ $H_{i} \cap \operatorname{Im}\left(\rho_{0,[r]}^{\{l\} \text {-geom }}\right)$ (see Lemma 1.6(ii)). Thus, $H_{i}$ fits into the following exact sequences: 


$$
\begin{gathered}
1 \longrightarrow H_{i}^{\text {geom }} \longrightarrow H_{i} \longrightarrow \operatorname{Im}\left(\rho_{0,3}^{\{l\}}\right) \longrightarrow 1 \\
1 \longrightarrow \operatorname{Im}\left(\rho_{0, r}^{\{l\}}\right) \longrightarrow H_{i} \longrightarrow Q_{i}\left(\subseteq \mathfrak{S}_{r}\right) \longrightarrow 1
\end{gathered}
$$

(Here, the surjectivity of $H_{i} \rightarrow \operatorname{Im}\left(\rho_{0,3}^{\{l\}}\right)$ follows from Lemma 1.6(i).) By the various definitions involved, this open subgroup $H_{i} \subseteq \operatorname{Im}\left(\rho_{0,[r]}^{\{l\}}\right)$ corresponds to the intermediate connected finite étale covering

$$
\left[\mathcal{M}_{0, r} / Q_{i}\right] \longrightarrow \mathcal{M}_{0,[r]}
$$

of the $\mathfrak{S}_{r}$-covering $\mathcal{M}_{0, r} \rightarrow\left[\mathcal{M}_{0, r} / \mathfrak{S}_{r}\right]=\mathcal{M}_{0,[r]}$, where $\left[\mathcal{M}_{0, r} /(-)\right]$ is the quotient of $\mathcal{M}_{0, r}$ by $(-)$ in the sense of stacks. Now we write

$$
\operatorname{Aut}_{k}^{Q_{1}, Q_{2}}\left(\mathcal{M}_{0, r}\right)
$$

for the set of automorphisms of $\mathcal{M}_{0, r}$ over $k$ which are compatible with the respective actions $Q_{1} \hookrightarrow \mathfrak{S}_{r} \rightarrow \operatorname{Aut}_{k}\left(\mathcal{M}_{0, r}\right)$ and $Q_{2} \hookrightarrow \mathfrak{S}_{r} \rightarrow \operatorname{Aut}_{k}\left(\mathcal{M}_{0, r}\right)$ relative to an isomorphism $Q_{1} \stackrel{\sim}{\rightarrow} Q_{2}$ of finite groups, that is, the subset of $\operatorname{Aut}_{k}\left(\mathcal{M}_{0, r}\right)$ consisting of automorphisms $\phi$ of $\mathcal{M}_{0, r}$ over $k$ which fit into a commutative diagram

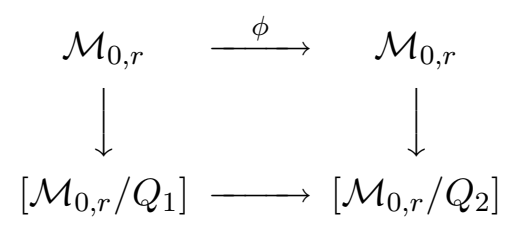

where the vertical arrows are natural morphisms and where the horizontal arrows are isomorphisms over $k$. Then we define a map

$$
\Phi: \operatorname{Aut}_{k}^{Q_{1}, Q_{2}}\left(\mathcal{M}_{0, r}\right) \longrightarrow \operatorname{Isom}_{\operatorname{Im}\left(\rho_{0,3}\right)}\left(H_{1}, H_{2}\right) / \operatorname{Inn}\left(H_{2}^{\text {geom }}\right)
$$

as follows. Let $\phi \in \operatorname{Aut}_{k}^{Q_{1}, Q_{2}}\left(\mathcal{M}_{0, r}\right)$. Then it follows from the definition of $\operatorname{Aut}_{k}^{Q_{1}, Q_{2}}\left(\mathcal{M}_{0, r}\right)$ that $\phi$ induces a diagram

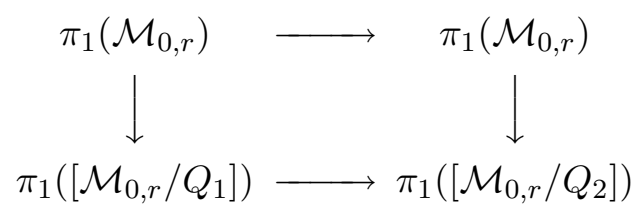

where the top horizontal arrow is the $\pi_{1}\left(\mathcal{M}_{0, r} \otimes_{k} \bar{k}\right)$-conjugacy class of the automorphism of $\pi_{1}\left(\mathcal{M}_{0, r}\right)$ induced by $\phi$, and this diagram commutes up to 
$\pi_{1}\left(\left[\mathcal{M}_{0, r} / Q_{2}\right] \otimes_{k} \bar{k}\right)$-inner automorphism. Thus, by considering the $H_{2}^{\text {geom }}$ conjugacy class of the isomorphism

$$
H_{1}=\pi_{1}\left(\left[\mathcal{M}_{0, r} / Q_{1}\right]\right) / \operatorname{Ker}\left(\rho_{0, r}^{\{l\}}\right) \stackrel{\sim}{\longrightarrow} \pi_{1}\left(\left[\mathcal{M}_{0, r} / Q_{2}\right]\right) / \operatorname{Ker}\left(\rho_{0, r}^{\{l\}}\right)=H_{2}
$$

induced by the lower horizontal arrow in the above diagram (note that by Lemma 4.2(i), the top horizontal arrow in the above diagram preserves $\left.\operatorname{Ker}\left(\rho_{0, r}^{\{l\}}\right) \subseteq \pi_{1}\left(\mathcal{M}_{0, r}\right)\right)$, we obtain an element $\Phi(\phi)$ of $\operatorname{Isom}_{\operatorname{Im}\left(\rho_{0,3}^{\{l\}}\right)}\left(H_{1}, H_{2}\right) /$ $\operatorname{Inn}\left(H_{2}^{\text {geom }}\right)$, as desired.

The purpose of Section 5 is to prove the surjectivity of this map $\Phi$ under the assumption that

$$
(*)^{\text {prime }}: l \text { is prime to the orders of } Q_{1} \text { and } Q_{2} \text {. }
$$

For the remainder of this section, suppose that the above condition $(*)$ prime is satisfied.

LEMMA 5.1 (preserving the $\mathcal{M}_{0, r}$-parts). Let $\phi: H_{1} \stackrel{\sim}{\rightarrow} H_{2}$ be an isomorphism over $\operatorname{Im}\left(\rho_{0,3}^{\{l\}}\right)$. Then $\phi\left(\operatorname{Im}\left(\rho_{0, r}^{\{l\} \text {-geom }}\right)\right)=\operatorname{Im}\left(\rho_{0, r}^{\{l\} \text {-geom }}\right)$. Moreover, if $k$ contains a primitive lth root of unity, then $\phi\left(\operatorname{Im}\left(\rho_{0, r}^{\{l\}}\right)\right)=\operatorname{Im}\left(\rho_{0, r}^{\{l\}}\right)$.

Proof. It follows immediately from Lemma 4.3(i), together with the assumption that the condition $(*)^{\text {prime }}$ is satisfied (see the discussion preceding Lemma 5.1), that $\operatorname{Im}\left(\rho_{0, r}^{\{l\} \text {-geom }}\right) \subseteq H_{i}^{\text {geom }}$ is the maximal pro- $l$ closed subgroup of $H_{i}^{\text {geom }}$; therefore, it follows that $\phi\left(\operatorname{Im}\left(\rho_{0, r}^{\{l\} \text {-geom }}\right)\right)=\operatorname{Im}\left(\rho_{0, r}^{\{l\} \text {-geom }}\right)$. Moreover, if $k$ contains a primitive $l$ th root of unity, then it follows from Lemma 4.3(ii), together with the assumption that the condition $(*)^{\text {prime }}$ is satisfied (see the discussion preceding Lemma 5.1), that $\operatorname{Im}\left(\rho_{0, r}^{\{l\}}\right) \subseteq H_{i}$ is the maximal pro- $l$ closed subgroup of $H_{i}$; therefore, it follows that $\phi\left(\operatorname{Im}\left(\rho_{0, r}^{\{l\}}\right)\right)=$ $\operatorname{Im}\left(\rho_{0, r}^{\{l\}}\right)$.

Next, we write

$$
\widetilde{\Phi}: \operatorname{Aut}_{k}^{Q_{1}, Q_{2}}\left(\mathcal{M}_{0, r}\right) \longrightarrow \operatorname{Isom}_{\operatorname{Im}\left(\rho_{0,3}^{\{l\}}\right)}\left(H_{1}, H_{2}\right) / \operatorname{Inn}\left(\operatorname{Im}\left(\rho_{0, r}^{\{l\} \text {-geom }}\right)\right)
$$

for the map defined as follows. Let $\phi \in \mathrm{Aut}_{k}^{Q_{1}, Q_{2}}\left(\mathcal{M}_{0, r}\right)$. Then $\phi$ determines an $\operatorname{Im}\left(\rho_{0, r}^{\{l\} \text {-geom }}\right)$-conjugacy class of an automorphism of $\operatorname{Im}\left(\rho_{0, r}^{\{l\}}\right)$ over $\operatorname{Im}\left(\rho_{0,3}^{\{l\}}\right)$. Moreover, by the definition of $\operatorname{Aut}_{k}^{Q_{1}, Q_{2}}\left(\mathcal{M}_{0, r}\right)$, this $\operatorname{Im}\left(\rho_{0, r}^{\{l\} \text {-geom }}\right)$ conjugacy class is compatible with the respective outer actions of $Q_{1}$ and $Q_{2}$ 
on $\operatorname{Im}\left(\rho_{0, r}^{\{l\}}\right)$ relative to an isomorphism $Q_{1} \stackrel{\sim}{\rightarrow} Q_{2}$. Therefore, $\operatorname{since} \operatorname{Im}\left(\rho_{0, r}^{\{l\}}\right)$ is

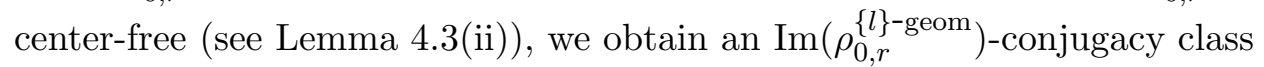
$\widetilde{\Phi}(\phi)$ of an isomorphism

$$
H_{1} \simeq \operatorname{Im}\left(\rho_{0, r}^{\{l\}}\right) \stackrel{\text { out }}{\rtimes} Q_{1} \stackrel{\sim}{\longrightarrow} \operatorname{Im}\left(\rho_{0, r}^{\{l\}}\right) \stackrel{\text { out }}{\rtimes} Q_{2} \simeq H_{2}
$$

(see the discussion on topological groups in Section 0 ) over $\operatorname{Im}\left(\rho_{0,3}^{\{l\}}\right)$.

Note that by the various definitions involved, the diagram

$$
\begin{gathered}
\operatorname{Aut}_{k}^{Q_{1}, Q_{2}}\left(\mathcal{M}_{0, r}\right) \stackrel{\widetilde{\Phi}}{\longrightarrow} \operatorname{Isom}_{\operatorname{Im}\left(\rho_{0,3}^{\{l\}}\right)}\left(H_{1}, H_{2}\right) / \operatorname{Inn}\left(\operatorname{Im}\left(\rho_{0, r}^{\{l\} \text {-geom }}\right)\right) \\
\|_{\operatorname{Aut}_{k}^{Q_{1}, Q_{2}}\left(\mathcal{M}_{0, r}\right) \stackrel{\Phi}{\longrightarrow}} \operatorname{Isom}_{\operatorname{Im}\left(\rho_{0,3}^{\{l\}}\right)}\left(H_{1}, H_{2}\right) / \operatorname{Inn}\left(H_{2}^{\text {geom }}\right)
\end{gathered}
$$

-where the right-hand vertical arrow is the natural surjection-commutes.

LEMMA 5.2 (a Grothendieck conjecture-type lemma for certain images of the universal monodromy). In the above diagram, the following hold:

(i) $\widetilde{\Phi}$ is injective,

(ii) $\widetilde{\Phi}$ is surjective,

(iii) $\Phi$ is surjective; moreover, for $\phi, \phi^{\prime} \in \operatorname{Aut}_{k}^{Q_{1}, Q_{2}}\left(\mathcal{M}_{0, r}\right)$, it holds that $\Phi(\phi)=\Phi\left(\phi^{\prime}\right)$ if and only if $\phi^{\prime} \circ \phi^{-1} \in \operatorname{Aut}_{k}\left(\mathcal{M}_{0, r}\right)$ is an element of the image of the composite $Q_{2} \hookrightarrow \mathfrak{S}_{r} \rightarrow \operatorname{Aut}\left(\mathcal{M}_{0, r}\right)$.

Proof. First, we consider assertion (i). To prove the injectivity of $\widetilde{\Phi}$-by replacing $k$ with a finite extension of $k$-we may assume without loss of generality that $k$ contains a primitive $l$ th root of unity (see Remark 5.2.1 below). Now we have a commutative diagram

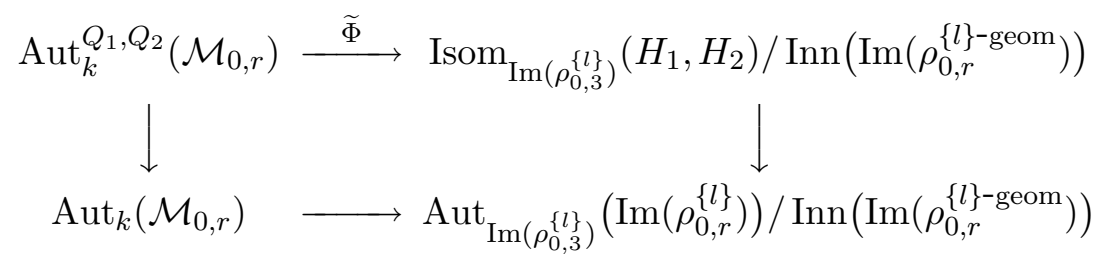

where the left-hand vertical arrow is the natural inclusion, where the righthand vertical arrow is the map obtained by restricting elements of $\operatorname{Isom}_{\operatorname{Im}\left(\rho_{0,3}^{\{l\}}\right)}\left(H_{1}, H_{2}\right) / \operatorname{Inn}\left(\operatorname{Im}\left(\rho_{0, r}^{\{l\} \text {-geom }}\right)\right)$ to $\operatorname{Im}\left(\rho_{0, r}^{\{l\}}\right) \subseteq H_{i}$ (see Lemma 5.1), 
and where the lower horizontal arrow is the homomorphism obtained in Lemma 4.2(i). Thus, since the lower horizontal arrow is injective (see Lemma 4.3 (iii)), it follows that $\widetilde{\Phi}$ is injective. This completes the proof of assertion (i).

Next, we consider assertion (ii). To prove the surjectivity of $\widetilde{\Phi}$, it follows from assertion (i), together with Galois descent, that by replacing $k$ with a finite extension of $k$, we may assume without loss of generality that $k$ contains a primitive $l$ th root of unity (see Remark 5.2 .1 below). Let $\phi: H_{1} \stackrel{\sim}{\rightarrow}$ $H_{2}$ be an isomorphism over $\operatorname{Im}\left(\rho_{0,3}^{\{l\}}\right)$. Then it follows from Lemma 5.1 that we obtain a commutative diagram

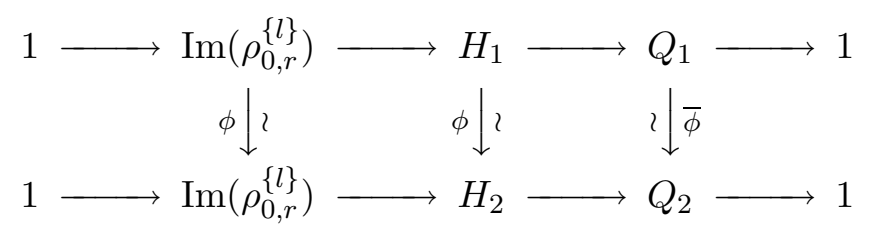

where the horizontal sequences are exact and where the vertical arrows are isomorphisms. It now follows from Lemma $4.3(\mathrm{iii})$ that the $\operatorname{Im}\left(\rho_{0, r}^{\{l\} \text {-geom }}\right)$ conjugacy class of the left-hand vertical arrow arises from an automorphism $\widetilde{\phi}$ of $\mathcal{M}_{0, r}$ over $k$. Moreover, since the above diagram commutes, it follows from assertion (i) that this automorphism $\widetilde{\phi}$ is compatible with the respective actions $Q_{1} \hookrightarrow \mathfrak{S}_{r} \rightarrow \operatorname{Aut}_{k}\left(\mathcal{M}_{0, r}\right)$ and $Q_{2} \hookrightarrow \mathfrak{S}_{r} \rightarrow \operatorname{Aut}_{k}\left(\mathcal{M}_{0, r}\right)$ relative to the isomorphism $\bar{\phi}: Q_{1} \stackrel{\sim}{\rightarrow} Q_{2}$, that is, $\widetilde{\phi}$ is an element of $\operatorname{Aut}_{k}^{Q_{1}, Q_{2}}\left(\mathcal{M}_{0, r}\right)$. This completes the proof of assertion (ii). Assertion (iii) follows immediately from assertions (i) and (ii), together with the various definitions involved.

REMARK 5.2.1. Let $\zeta_{l} \in \bar{k}$ be a primitive $l$ th root of unity. Then it follows from $\left[2\right.$, Theorems A, B] that $\operatorname{Ker}\left(G_{k} \rightarrow \operatorname{Im}\left(\rho_{0,3}^{\{l\}}\right)\right) \subseteq G_{k\left(\zeta_{l}\right)}$. Therefore, if we write

$$
\left(H_{i}\right)_{k\left(\zeta_{l}\right)}=H_{i} \cap \rho_{0,[r]}^{\{l\}}\left(\pi_{1}\left(\mathcal{M}_{0,[r]} \otimes_{k} k\left(\zeta_{l}\right)\right)\right) \subseteq \operatorname{Im}\left(\rho_{0,[r]}^{\{l\}}\right)
$$

and

$$
\left(H_{i}\right)_{k\left(\zeta_{l}\right)}^{\text {geom def }}=\left(H_{i}\right)_{k\left(\zeta_{l}\right)} \cap H_{i}^{\text {geom }}
$$

then $\left(H_{i}\right)_{k\left(\zeta_{l}\right)}^{\text {geom }}=H_{i}^{\text {geom }}$. In particular, $\left(H_{i}\right)_{k\left(\zeta_{l}\right)}$ fits into similar exact sequences

$$
\begin{gathered}
1 \longrightarrow\left(H_{i}\right)_{k\left(\zeta_{l}\right)}^{\text {geom }}\left(=H_{i}^{\text {geom }}\right) \longrightarrow\left(H_{i}\right)_{k\left(\zeta_{l}\right)} \longrightarrow \rho_{0,3}^{\{l\}}\left(\pi_{1}\left(\mathcal{M}_{0,3} \otimes_{k} k\left(\zeta_{l}\right)\right)\right) \longrightarrow 1, \\
1 \longrightarrow \rho_{0, r}^{\{l\}}\left(\pi_{1}\left(\mathcal{M}_{0, r} \otimes_{k} k\left(\zeta_{l}\right)\right)\right) \longrightarrow\left(H_{i}\right)_{k\left(\zeta_{l}\right)} \longrightarrow Q_{i}\left(\subseteq \mathfrak{S}_{r}\right) \longrightarrow 1,
\end{gathered}
$$


to the exact sequences

$$
\begin{gathered}
1 \longrightarrow H_{i}^{\text {geom }} \longrightarrow H_{i} \longrightarrow \operatorname{Im}\left(\rho_{0,3}^{\{l\}}\right) \longrightarrow 1 \\
1 \longrightarrow \operatorname{Im}\left(\rho_{0, r}^{\{l\}}\right) \longrightarrow H_{i} \longrightarrow Q_{i}\left(\subseteq \mathfrak{S}_{r}\right) \longrightarrow 1
\end{gathered}
$$

\section{$\S 6$. Proof of the main result}

In this section, we prove that the isomorphism class of an $l$-monodromically full hyperbolic curve of genus zero over a finitely generated field of characteristic zero is completely determined by the kernel of the associated pro-l outer Galois representation (see Theorem 6.1 below).

Theorem 6.1 (Galois-theoretic characterization of isomorphism classes of monodromically full hyperbolic curves of genus zero). Let $l$ be a prime number, let $k$ be a finitely generated field of characteristic zero (see the discussion on numbers in Section 0), let $\bar{k}$ be an algebraic closure of $k$, let $G_{k} \stackrel{\text { def }}{=} \operatorname{Gal}(\bar{k} / k)$, and let $X_{1}=\left(C_{1}, D_{1} \subseteq C_{1}\right), X_{2}=\left(C_{2}, D_{2} \subseteq C_{2}\right)$ be hyperbolic curves (see Definition 1.1(ii)) of genus zero over $k$ which are l-monodromically full (see Definition 2.2(i)). Suppose that the following condition $(\dagger)^{\text {prime }}$ is satisfied:

$(\dagger)^{\text {prime }}$ : There exists a finite Galois extension $k^{\prime} \subseteq \bar{k}$ of $k$ of degree prime to

$l$ such that $X_{1} \otimes_{k} k^{\prime}$ and $X_{2} \otimes_{k} k^{\prime}$ are split (see Definition 1.5(i)).

(For example, if one of the following conditions is satisfied, then the above condition $(\dagger)^{\text {prime }}$ is satisfied:

- $X_{1}$ and $X_{2}$ are split;

- if we write $r_{i}$ for the number of cusps of $X_{i}$ that is, if $X_{i}$ is of type $\left(0, r_{i}\right)$, then $l$ is prime to $r_{1}$ ! and $r_{2}$ ! or, equivalently, $r_{1}, r_{2}<l$.)

Then the following conditions are equivalent:

(i) $X_{1}$ is isomorphic to $X_{2}$ over $k$;

(ii) for $i=1,2$, write

$$
\rho_{X_{i} / k}^{\{l l\}}: G_{k} \longrightarrow \operatorname{Out}\left(\pi_{1}\left(\left(C_{i} \backslash D_{i}\right) \otimes_{k} \bar{k}\right)^{(l)}\right)
$$

for the pro-l outer Galois representation associated to $X_{i}$; then $\operatorname{Ker}\left(\rho_{X_{1} / k}^{\{l\}}\right)=\operatorname{Ker}\left(\rho_{X_{2} / k}^{\{l\}}\right)$.

Proof. The implication

$$
\text { (i) } \Longrightarrow(\mathrm{ii})
$$


is immediate; thus, to verify Theorem 6.1 , it suffices to show the implication

$$
\text { (ii) } \Longrightarrow(\mathrm{i}) \text {. }
$$

Suppose that condition (ii) is satisfied. We write $N \stackrel{\text { def }}{=} \operatorname{Ker}\left(\rho_{X_{1} / k}^{\{l\}}\right)=$ $\operatorname{Ker}\left(\rho_{X_{2} / k}^{\{l\}}\right) \subseteq G_{k}$ (see (ii)), and we write $r_{i}$ for the number of cusps of the hyperbolic curve $X_{i}$; that is, $X_{i}$ is a hyperbolic curve of type $\left(0, r_{i}\right)$.

It now follows immediately that the bijection of $\operatorname{sets} \phi: \operatorname{Im}\left(\rho_{X_{1} / k}^{\{l\}}\right) \rightarrow$ $\operatorname{Im}\left(\rho_{X_{2} / k}^{\{l\}}\right)$ obtained as the composite

$$
\operatorname{Im}\left(\rho_{X_{1} / k}^{\{l\}}\right) \stackrel{\sim}{\longleftarrow} G_{k} / N \stackrel{\sim}{\longrightarrow} \operatorname{Im}\left(\rho_{X_{2} / k}^{\{l\}}\right),
$$

where the $\stackrel{\sim}{\leftarrow}$ and $\stackrel{\sim}{\rightarrow}$ are natural isomorphisms, is an isomorphism of profinite groups. Moreover, it follows from Lemma 1.6(ii) that this isomorphism $\phi$ is an isomorphism over $\operatorname{Im}\left(\rho_{0,3}^{\{l\}}\right)$. Thus, since $X_{1}$ and $X_{2}$ are $l$-monodromically full and since the condition $(\dagger)^{\text {prime }}$ is satisfied, it follows from an argument similar to that used in the proof of Lemma 5.1 (see condition $(*)^{\text {prime }}$ in the discussion preceding Lemma 5.1) that $\phi \operatorname{maps} \operatorname{Im}\left(\rho_{0, r_{1}}^{\{l\} \text {-geom }}\right) \subseteq \operatorname{Im}\left(\rho_{X_{1} / k}^{\{l\}}\right)$ bijectively onto $\operatorname{Im}\left(\rho_{0, r_{2}}^{\{l\} \text {-geom }}\right) \subseteq \operatorname{Im}\left(\rho_{X_{2} / k}^{\{l\}}\right)$. In particular, it follows immediately from Lemma $4.2(\mathrm{ii})$ that $r_{1}=r_{2}$.

Write $r \stackrel{\text { def }}{=} r_{1}=r_{2}, Q_{i}$ for the image of the composite $\operatorname{Im}\left(\rho_{X_{i} / k}^{\{l\}}\right) \hookrightarrow$ $\operatorname{Im}\left(\rho_{0,[r]}^{\{l\}}\right) \rightarrow \operatorname{Im}\left(\rho_{0,[r]}^{\{l\}}\right) / \operatorname{Im}\left(\rho_{0, r}^{\{l\}}\right)\left(\simeq \mathfrak{S}_{r} ;\right.$ see Lemma 1.6(iii)), and write $\left[\mathcal{M}_{0, r} /\right.$ $\left.Q_{i}\right] \rightarrow \mathcal{M}_{0,[r]}$ for the intermediate connected finite étale covering of the $\mathfrak{S}_{r^{-}}$ covering $\mathcal{M}_{0, r} \rightarrow\left[\mathcal{M}_{0, r} / \mathfrak{S}_{r}\right]=\mathcal{M}_{0,[r]}$ corresponding to the image $\operatorname{Im}\left(\rho_{X_{i} / k}^{\{l\}}\right) \subseteq \operatorname{Im}\left(\rho_{0,[r]}^{\{l\}}\right)$. Then it follows from Lemma 5.2(iii), together with the assumption that the condition $(\dagger)^{\text {prime }}$ is satisfied (see condition $(*)^{\text {prime }}$ in the discussion preceding Lemma 5.1), that the isomorphism obtained as the composite

$$
\begin{gathered}
\pi_{1}\left(\left[\mathcal{M}_{0, r} / Q_{1}\right]\right) / \operatorname{Ker}\left(\rho_{0, r}^{\{l\}}\right)=\operatorname{Im}\left(\rho_{X_{1} / k}^{\{l\}}\right) \\
\stackrel{\phi}{\sim} \operatorname{Im}\left(\rho_{X_{2} / k}^{\{l\}}\right)=\pi_{1}\left(\left[\mathcal{M}_{0, r} / Q_{2}\right]\right) / \operatorname{Ker}\left(\rho_{0, r}^{\{l\}}\right)
\end{gathered}
$$

arises from the lower horizontal arrow in a commutative diagram

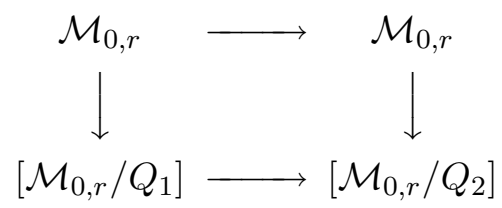


where the vertical arrows are natural morphisms and where the horizontal arrows are isomorphisms over $k$. Therefore, it follows from Lemma 4.1(ii), together with the various definitions involved, that if we write $\widetilde{s}_{X_{i}} \in$ $\mathcal{M}_{0,[r]}(k)$ for the classifying morphism of $X_{i}$, then the elements of

$$
\operatorname{Hom}_{\operatorname{Im}\left(\rho_{0,3}^{\{1\}}\right)}\left(G_{k}, \operatorname{Im}\left(\rho_{0,[r]}^{\{l\}}\right)\right) / \operatorname{Inn}\left(\operatorname{Im}\left(\rho_{0,[r]}^{\{l\} \text {-geom }}\right)\right),
$$

determined by $\widetilde{s}_{X_{1}}$ and $\widetilde{s}_{X_{2}}$, respectively, coincide. Thus, it follows from Lemma $4.3(\mathrm{v})$ that $X_{1}$ is isomorphic to $X_{2}$ over $k$, as desired. This completes the proof of the above implication.

\section{$\S 7$. Example I: Hyperbolic curves of type $(0,4)$ over number fields}

In this section, we consider the monodromic fullness of hyperbolic curves of type $(0,4)$ over number fields. In particular, we obtain sufficient conditions for such a hyperbolic curve to be monodromically full (see Theorem 7.9 and Corollaries 7.11 and 7.12 below). Moreover, as an application of these sufficient conditions, we obtain a Galois-theoretic characterization of the isomorphism classes of certain hyperbolic curves of type $(0,4)$ over number fields (see Corollary 7.13 below). Here, suppose that $k$ is a number field (see the discussion on numbers in Section 0 ), let $\mathfrak{o}_{k} \subseteq k$ be the ring of integers of $k$, and let $\lambda \in k \backslash\{0,1\}$ be an element of $k \backslash\{0,1\}$. Moreover, in this section, if $k^{\prime} \subseteq \bar{k}$ is a finite extension of $k$ and if $p$ is a prime number, then write $\mathfrak{P}\left(k^{\prime} ; p\right)$ for the set of nonarchimedean primes $\mathfrak{p}$ of $k^{\prime}$ such that the residue characteristic of $\mathfrak{p}$ is $p$.

Definition 7.1. Let $l$ be an odd prime number, and let $\zeta_{l} \in \bar{k}$ be a primitive $l$ th root of unity. We have the following.

(i) We write $k_{l} \subseteq \bar{k}$ for the Galois extension of $k\left(\zeta_{l}\right)$ corresponding to the quotient

$$
G_{k\left(\zeta_{l}\right)} \rightarrow \rho_{0,3}^{\{l\}}\left(G_{k\left(\zeta_{l}\right)}\right) \rightarrow \rho_{0,3}^{\{l\}}\left(G_{k\left(\zeta_{l}\right)}\right)^{\mathrm{ab}} \otimes_{\mathbb{Z}_{l}} \mathbb{F}_{l} .
$$

(ii) We write $\pi_{1}\left(\mathbb{P}_{k\left(\zeta_{l}\right)}^{1} \backslash\{0,1, \infty\}\right) \rightarrow Q_{l}$ for the quotient of $\pi_{1}\left(\mathbb{P}_{k\left(\zeta_{l}\right)}^{1} \backslash\right.$ $\{0,1, \infty\})$ obtained as the composite

$$
\begin{gathered}
\pi_{1}\left(\mathbb{P}_{k\left(\zeta_{l}\right)}^{1} \backslash\{0,1, \infty\}\right) \simeq \pi_{1}\left(\mathcal{M}_{0,4} \otimes_{k} k\left(\zeta_{l}\right)\right) \rightarrow \rho_{0,4}^{\{l\}}\left(\pi_{1}\left(\mathcal{M}_{0,4} \otimes_{k} k\left(\zeta_{l}\right)\right)\right) \\
\rightarrow \rho_{0,4}^{\{l\}}\left(\pi_{1}\left(\mathcal{M}_{0,4} \otimes_{k} k\left(\zeta_{l}\right)\right)\right)^{\mathrm{ab}} \otimes_{\mathbb{Z}_{l}} \mathbb{F}_{l},
\end{gathered}
$$

where the first arrow is the outer isomorphism obtained by an isomorphism $\mathbb{P}_{k}^{1} \backslash\{0,1, \infty\} \simeq \mathcal{M}_{0,4}$ over $k$ (see Lemma $4.1(\mathrm{i})$ ). 
(iii) We write

$$
\begin{gathered}
X_{l} \stackrel{\text { def }}{=} \operatorname{Spec} k\left[s^{ \pm 1}, t^{ \pm 1}\right] /\left(s^{l}+t^{l}-1\right) \\
\rightarrow \operatorname{Spec} k\left[u^{ \pm 1}, 1 /(u-1)\right]=\mathbb{P}_{k}^{1} \backslash\{0,1, \infty\},
\end{gathered}
$$

where $s, t$, and $u$ are indeterminates, for the connected finite étale covering of $\mathbb{P}_{k}^{1} \backslash\{0,1, \infty\}$ determined by the homomorphism of algebras over $k$

$$
\begin{aligned}
k\left[u^{ \pm 1}, 1 /(u-1)\right] & \longrightarrow k\left[s^{ \pm 1}, t^{ \pm 1}\right] /\left(s^{l}+t^{l}-1\right) \\
u & \mapsto s^{l} .
\end{aligned}
$$

LEMMA 7.2 (properties of certain extensions). Let $l$ be an odd prime number, and let $\zeta_{l} \in \bar{k}$ be a primitive lth root of unity. Then $k_{l}$ (see Definition 7.1(i)) is a finite abelian extension of $k\left(\zeta_{l}\right)$ of degree a power of $l$. Moreover, the extension $k_{l}$ of $k$ is unramified outside $\mathfrak{P}(k ; l)$. In particular, $Q_{l}$ (see Definition 7.1(ii)) is a finite group.

Proof. It follows from Lemma 2.5 (resp., Lemma 4.3(ii)) that the quotient

$$
G_{k\left(\zeta_{l}\right)} \rightarrow \rho_{0,3}^{\{l\}}\left(G_{k\left(\zeta_{l}\right)}\right)
$$

is topologically finitely generated (resp., pro- $l$ ). Moreover, it follows from $\left[2\right.$, Theorems A, B] that the algebraic extension of $k\left(\zeta_{l}\right)$ corresponding to the above quotient is unramified outside $\mathfrak{P}\left(k\left(\zeta_{l}\right) ; l\right)$. Therefore, Lemma 7.2 follows immediately from the fact that the extension $k\left(\zeta_{l}\right)$ of $k$ is unramified outside $\mathfrak{P}(k ; l)$. This completes the proof of Lemma 7.2.

LEMMA 7.3 (properties of certain coverings). Let $l$ be an odd prime number, and let $\zeta_{l} \in \bar{k}$ be a primitive lth root of unity. Then

$$
\pi_{1}\left(\mathcal{M}_{0,4}\right) \stackrel{\{l\}}{\stackrel{\text { def }}{=}} \pi_{1}\left(\mathcal{M}_{0,4}\right) / \operatorname{Ker}\left(\rho_{0,4}^{\{l\} \text {-geom }}\right)
$$

(note that since $\pi_{1}\left(\mathcal{M}_{0,4} \otimes_{k} \bar{k}\right)$ is normal in $\pi_{1}\left(\mathcal{M}_{0,4}\right)$ and since $\operatorname{Ker}\left(\rho_{0,4}^{\{l\} \text {-geom }}\right)$ is characteristic in $\pi_{1}\left(\mathcal{M}_{0,4} \otimes_{k} \bar{k}\right)$ [see Lemma 4.2(i)], it holds that $\operatorname{Ker}\left(\rho_{0,4}^{\{l\} \text {-geom }}\right)$ is normal in $\left.\pi_{1}\left(\mathcal{M}_{0,4}\right)\right)$ and that

$$
\rho \frac{\{l\}}{0,4}: \pi_{1}\left(\mathcal{M}_{0,4}\right) \stackrel{\{l\}}{\longrightarrow} \operatorname{Out}\left(\Delta_{0,4}^{\{l\}}\right)
$$

for the homomorphism induced by $\rho_{0,4}^{\{l\}}: \pi_{1}\left(\mathcal{M}_{0,4}\right) \rightarrow \operatorname{Out}\left(\Delta_{0,4}^{\{l\}}\right)$. Fix an isomorphism $\mathcal{M}_{0,4} \simeq \mathbb{P}_{k}^{1} \backslash\{0,1, \infty\}$ over $k$ (see Lemma 4.1(i)). Then the following hold. 


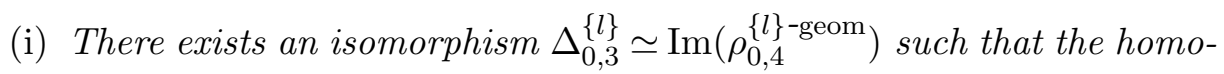
morphism

$$
G_{k} \longrightarrow \operatorname{Out}\left(\operatorname{Im}\left(\rho_{0,4}^{\{l\} \text {-geom }}\right)\right) \simeq \operatorname{Out}\left(\Delta_{0,3}^{\{l\}}\right)
$$

determined by the natural exact sequence of profinite groups

$$
1 \longrightarrow \operatorname{Im}\left(\rho_{0,4}^{\{l\} \text {-geom }}\right) \longrightarrow \pi_{1}\left(\mathcal{M}_{0,4}\right) \stackrel{\{l\}}{\longrightarrow} \longrightarrow G_{k} \longrightarrow 1
$$

coincides with $\rho_{0,3}^{\{l\}}$.

(ii) The finite étale covering $X_{l} \rightarrow \mathcal{M}_{0,4}$ (see Definition 7.1 (iii)) arises from an open subgroup of $\pi_{1}\left(\mathcal{M}_{0,4}\right) \frac{\{l\}}{2}$; that is, if we write $\pi_{1}\left(X_{l} \otimes_{k}\right.$ $\bar{k})^{\{l\}}$ for the maximal pro-l quotient of $\pi_{1}\left(X_{l} \otimes_{k} \bar{k}\right)$ and write $\pi_{1}\left(X_{l}\right) \underline{\{l\}}$ for the quotient of $\pi_{1}\left(X_{l}\right)$ by the kernel of the natural surjection $\pi_{1}\left(X_{l} \otimes_{k} \bar{k}\right) \rightarrow \pi_{1}\left(X_{l} \otimes_{k} \bar{k}\right)^{\{l\}}$, then the finite étale covering $X_{l} \rightarrow \mathcal{M}_{0,4}$ induces a commutative diagram of profinite groups

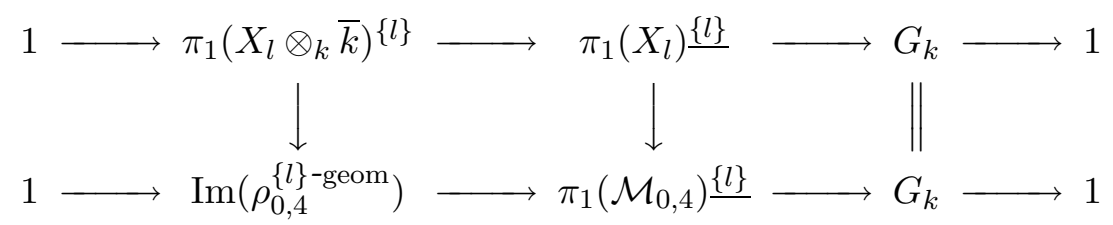

where the horizontal sequences are exact and where the vertical arrows are open injections.

(iii) The image of the left-hand vertical arrow in the diagram in assertion (ii) coincides with the kernel of the natural surjection $\operatorname{Im}\left(\rho_{0,4}^{\{l\} \text {-geom }}\right) \rightarrow \operatorname{Im}\left(\rho_{0,4}^{\{l\} \text {-geom }}\right)^{\text {ab }} \otimes_{\mathbb{Z}_{l}} \mathbb{F}_{l}$. In particular, $\pi_{1}\left(X_{l} \otimes_{k} \bar{k}\right)^{\{l\}} \subseteq$ $\operatorname{Im}\left(\rho_{0,4}^{\{l\} \text {-geom }}\right)$ is characteristic in $\operatorname{Im}\left(\rho_{0,4}^{\{l\} \text {-geom }}\right)$.

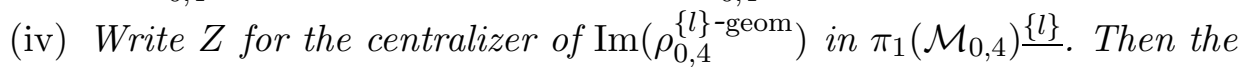
image of $Z$ in $G_{k}$ coincides with the kernel $\operatorname{Ker}\left(\rho_{0,3}^{\{l\}}\right)$ of $\rho_{0,3}^{\{l\}}$. Moreover, $Z=\operatorname{Ker}\left(\rho \frac{\{l\}}{0,4}\right)$.

(v) Write $Z_{X} \stackrel{\text { def }}{=} Z \cap \pi_{1}\left(X_{l}\right) \stackrel{\{l\}}{\subseteq} \subseteq \pi_{1}\left(X_{l}\right) \underline{\{l\}}$. Then $Z_{X}$ coincides with the centralizer of $\pi_{1}\left(X_{l} \otimes_{k} \bar{k}\right)^{\{l\}}$ in $\pi_{1}\left(X_{l}\right) \underline{\{l\}}$.

(vi) The kernel $\operatorname{Ker}\left(\rho \frac{\{l\}}{0,4}\right)$ of $\rho \frac{\{l\}}{0,4}$ is contained in $\pi_{1}\left(X_{l}\right) \frac{\{l\}}{}$, hence also in $\pi_{1}\left(X_{l}\right) \stackrel{\{l\}}{ } \times_{G_{k}} G_{k_{l}}$.

(vii) The finite étale covering $X_{l} \otimes_{k} k_{l} \rightarrow \mathcal{M}_{0,4} \otimes_{k} k\left(\zeta_{l}\right)$ is the connected finite étale covering of $\mathcal{M}_{0,4} \otimes_{k} k\left(\zeta_{l}\right)$ corresponding to the kernel of the natural surjection $\pi_{1}\left(\mathcal{M}_{0,4} \otimes_{k} k\left(\zeta_{l}\right)\right) \rightarrow Q_{l}$ (see Definition \%.1(ii)). 
Proof. Assertion (i) follows immediately from Lemmas 4.1(i) and 4.2(i), together with the definition of $\rho_{0,3}^{\{l\}}$. Assertions (ii) and (iii) follow immediately from the definition of the finite étale covering $X_{l} \rightarrow \mathcal{M}_{0,4}$, together with the well-known structure of the fundamental group of $\mathcal{M}_{0,4} \otimes_{k} \bar{k} \simeq$ $\mathbb{P}_{\bar{k}} \backslash\{0,1, \infty\}$. Next, we verify assertion (iv). The fact that the image of $Z$ in $G_{k}$ coincides with the kernel $\operatorname{Ker}\left(\rho_{0,3}^{\{l\}}\right)$ follows immediately from assertion (i). Moreover, it follows immediately from Lemma 1.6(i) that the image of $\operatorname{Ker}\left(\rho \frac{\{l\}}{0,4}\right) \subseteq \pi_{1}\left(\mathcal{M}_{0,4}\right) \frac{\{l\}}{\underline{ }}$ in $G_{k}$ coincides with the kernel $\operatorname{Ker}\left(\rho_{0,3}^{\{l\}}\right)$. Therefore, since $\operatorname{Im}\left(\rho_{0,4}^{\{l\} \text {-geom }}\right) \cap Z=\{1\}$ by Lemma 4.3 (i), to verify the fact that $Z=\operatorname{Ker}\left(\rho \frac{\{l\}}{0,4}\right)$ it suffices to verify that $\operatorname{Ker}\left(\rho_{0,4}^{\{l\}} \subseteq \subseteq\right.$. On the other hand, since $\operatorname{Im}\left(\rho_{0,4}^{\{l\} \text {-geom }}\right)$ is center-free, again by Lemma $4.3(\mathrm{i})$, one may easily verify that $Z \subseteq \pi_{1}\left(\mathcal{M}_{0,4}\right) \frac{\{l\}}{\underline{ }}$ is the maximal normal closed subgroup $N$ of $\pi_{1}\left(\mathcal{M}_{0,4}\right) \underline{\{l\}}$ such that $\operatorname{Im}\left(\rho_{0,4}^{\{l\} \text {-geom }}\right) \cap N=\{1\}$. Therefore, since $\operatorname{Im}\left(\rho_{0,4}^{\{l\} \text {-geom }}\right) \cap \operatorname{Ker}\left(\rho_{0,4}^{\{l\}}\right)=\{1\}$ by the definitions of the quotient $\pi_{1}\left(\mathcal{M}_{0,4}\right) \underline{\{l\}}$ and the homomorphism $\rho \frac{\{l\}}{0,4}$, it holds that $\operatorname{Ker}\left(\rho_{0,4}^{\{l\}} \subseteq Z\right.$. This completes the proof of assertion (iv). Next, we verify assertion (v). Observe that it is immediate that $Z_{X}$ is contained in the centralizer of $\pi_{1}\left(X_{l} \otimes_{k} \bar{k}\right)^{\{l\}}$ in $\pi_{1}\left(X_{l}\right) \underline{\{l\}}$. It now follows from assertion (iii) that we have a natural homomorphism

$$
\begin{aligned}
\phi: \operatorname{Aut}\left(\operatorname{Im}\left(\rho_{0,4}^{\{l\} \text {-geom }}\right)\right) & \longrightarrow \operatorname{Aut}\left(\pi_{1}\left(X_{l} \otimes_{k} \bar{k}\right)^{\{l\}}\right) \\
\alpha & \left.\mapsto \alpha\right|_{\pi_{1}\left(X_{l} \otimes_{k} \bar{k}\right)^{\{l\}} .}
\end{aligned}
$$

On the other hand, conjugation by elements of $\operatorname{Im}\left(\rho_{0,4}^{\{l\} \text {-geom }}\right)$ determines a homomorphism

$$
\iota: \operatorname{Im}\left(\rho_{0,4}^{\{l\} \text {-geom }}\right) \longrightarrow \operatorname{Aut}\left(\pi_{1}\left(X_{l} \otimes_{k} \bar{k}\right)^{\{l\}}\right),
$$

which is injective (see Lemma 4.3(i)). Now one may easily verify that the above homomorphism $\phi$ determines an isomorphism of $\operatorname{Aut}\left(\operatorname{Im}\left(\rho_{0,4}^{\{l\} \text {-geom }}\right)\right)$ with the normalizer of the image of the injection $\iota$ in $\operatorname{Aut}\left(\pi_{1}\left(X_{l} \otimes_{k} \bar{k}\right)^{\{l\}}\right)$. In particular, the above homomorphism $\phi$ is injective. Therefore, it follows immediately that the centralizer of $\pi_{1}\left(X_{l} \otimes_{k} \bar{k}\right)^{\{l\}}$ in $\pi_{1}\left(X_{l}\right) \underline{\{l\}}$ is contained in $Z_{X}$. This completes the proof of assertion (v). Next, we verify assertion (vi). It follows immediately from assertion (iv) that, to verify assertion (vi), it suffices to verify that $Z=Z_{X}$. On the other hand, it follows immediately 
from assertions (iv) and (v), together with the various definitions involved, that the image of $Z$ (resp., $Z_{X}$ ) in $G_{k}$ coincides with the kernel of the homomorphism $\rho_{0,3}^{\{l\}}$ (resp., $\rho: G_{k} \rightarrow \operatorname{Out}\left(\pi_{1}\left(X_{l} \otimes_{k} \bar{k}\right)^{\{l\}}\right)$ determined by the top horizontal sequence of the diagram in assertion (ii)). Therefore, it follows immediately from the fact that $Z_{X} \cap \pi_{1}\left(X_{l} \otimes_{k} \bar{k}\right)^{\{l\}} \subseteq Z \cap \operatorname{Im}\left(\rho_{0,4}^{\{l\} \text {-geom }}\right)=\{1\}$ (see Lemma 4.3(i)) that, to verify assertion (vi), it suffices to verify that $\operatorname{Ker}\left(\rho_{0,3}^{\{l\}}\right)=\operatorname{Ker}(\rho)$. The rest of the proof of assertion (vi) is devoted to proving that $\operatorname{Ker}\left(\rho_{0,3}^{\{l\}}\right)=\operatorname{Ker}(\rho)$ or, equivalently, that $\operatorname{Ker}\left(\rho_{0,3}^{\{l\}}\right) \subseteq \operatorname{Ker}(\rho)$ (see $[8$,

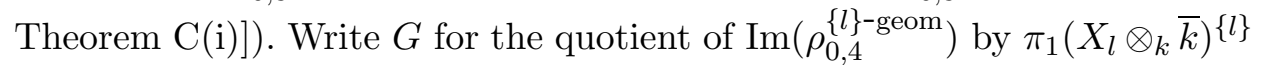
(i.e., $G=\operatorname{Gal}\left(X_{l} \otimes_{k} \bar{k} / \mathcal{M}_{0,4} \otimes_{k} \bar{k}\right)$; see assertion (iii)), and write

$$
\rho_{G}: G \longrightarrow \operatorname{Out}\left(\pi_{1}\left(X_{l} \otimes_{k} \bar{k}\right)^{\{l\}}\right)
$$

for the homomorphism determined by the natural exact sequence

$$
1 \longrightarrow \pi_{1}\left(X_{l} \otimes_{k} \bar{k}\right)^{\{l\}} \longrightarrow \operatorname{Im}\left(\rho_{0,4}^{\{l\} \text {-geom }}\right) \longrightarrow G \longrightarrow 1 \text {. }
$$

Then it follows immediately from the various definitions involved that $\rho\left(\operatorname{Ker}\left(\rho_{0,3}^{\{l\}}\right)\right) \subseteq \operatorname{Im}\left(\rho_{G}\right)$. On the other hand, it follows immediately from the definition of the finite étale covering $X_{l} \rightarrow \mathcal{M}_{0,4}$, together with the fact that $\operatorname{Ker}\left(\rho_{0,3}^{\{l\}}\right) \subseteq G_{k\left(\zeta_{l}\right)}$ (see Remark 5.2.1), that the action of $\operatorname{Im}\left(\rho_{G}\right)$ (resp., $\left.\rho\left(\operatorname{Ker}\left(\rho_{0,3}^{\{l\}}\right)\right)\right)$ on the set of conjugacy classes of cuspidal inertia subgroups of $\pi_{1}\left(X_{l} \otimes_{k} \bar{k}\right)^{\{l\}}$ is faithful (resp., trivial) (see Remark 1.3.1). Thus, it holds that $\rho\left(\operatorname{Ker}\left(\rho_{0,3}^{\{l\}}\right)\right)=\{1\}$, that is, that $\operatorname{Ker}\left(\rho_{0,3}^{\{l\}}\right) \subseteq \operatorname{Ker}(\rho)$. This completes the proof of assertion (vi). Assertion (vii) follows immediately from assertions (iii) and (vi), together with Lemma 1.6(i). This completes the proof of Lemma 7.3 .

LEMMA 7.4 (fibers and monodromic fullness). Let $l$ be an odd prime number, and let $\zeta_{l} \in \bar{k}$ be a primitive lth root of unity. Then the following four conditions are equivalent.

(i) The fiber of $X_{l} \otimes_{k} k_{l} \rightarrow \mathbb{P}_{k}^{1} \backslash\{0,1, \infty\}$ at the (image of the) $k$-rational point of $\mathbb{P}_{k}^{1} \backslash\{0,1, \infty\}$ corresponding to the element $\lambda \in k \backslash\{0,1\}$ is connected.

(ii) The composite

$$
G_{k\left(\zeta_{l}\right)} \longrightarrow \pi_{1}\left(\mathbb{P}_{k\left(\zeta_{l}\right)}^{1} \backslash\{0,1, \infty\}\right) \longrightarrow Q_{l},
$$


where the first arrow is the homomorphism (which is determined up to $\pi_{1}\left(\mathbb{P}_{\bar{k}} \backslash\{0,1, \infty\}\right)$-inner automorphism) induced by the $k\left(\zeta_{l}\right)$-rational point of $\mathbb{P}_{k\left(\zeta_{l}\right)}^{1} \backslash\{0,1, \infty\}$ corresponding to the element $\lambda \in k \backslash\{0,1\} \subseteq$ $k\left(\zeta_{l}\right) \backslash\{0,1\}$, is surjective.

(iii) The composite

$$
G_{k} \longrightarrow \pi_{1}\left(\mathbb{P}_{k}^{1} \backslash\{0,1, \infty\}\right) \stackrel{\sim}{\longrightarrow} \pi_{1}\left(\mathcal{M}_{0,4}\right) \longrightarrow \operatorname{Im}\left(\rho_{0,4}^{\{l\}}\right),
$$

where the first arrow is the homomorphism (which is determined up to $\pi_{1}\left(\mathbb{P}_{\bar{k}}^{1} \backslash\{0,1, \infty\}\right)$-inner automorphism) induced by the $k$-rational point of $\mathbb{P}_{k}^{1} \backslash\{0,1, \infty\}$ corresponding to the element $\lambda \in k \backslash\{0,1\}$ and where the second arrow is the outer isomorphism over $G_{k}$ obtained by an isomorphism $\mathbb{P}_{k}^{1} \backslash\{0,1, \infty\} \simeq \mathcal{M}_{0,4}$ over $k$ (see Lemma 4.1(i)), is surjective.

(iv) The hyperbolic curve $\left(\mathbb{P}_{k}^{1},\{0,1, \lambda, \infty\} \subseteq \mathbb{P}_{k}^{1}\right)$ of type $(0,4)$ over $k$ is $l$ monodromically full.

Proof. The equivalence

$$
\text { (i) } \Longleftrightarrow \text { (ii) }
$$

follows immediately from Lemma 7.3(vii), together with the various definitions involved. The implication

$$
\text { (iii) } \Longrightarrow(\text { ii) }
$$

follows immediately from Lemma 1.6(i), together with the fact that $\operatorname{Ker}\left(\rho_{0,3}^{\{l\}}\right) \subseteq G_{k\left(\zeta_{l}\right)}$ (see Remark 5.2.1). The equivalence

$$
\text { (iii) } \Longleftrightarrow \text { (iv) }
$$

follows immediately from the various definitions involved. Finally, we verify the implication

$$
\text { (ii) } \Longrightarrow \text { (iii). }
$$

Suppose that condition (ii) is satisfied. It follows from the fact that $\operatorname{Ker}\left(\rho_{0,3}^{\{l\}}\right) \subseteq G_{k\left(\zeta_{l}\right)}$ (see Remark 5.2.1) that, to verify condition (iii)-by replacing $k$ with $k\left(\zeta_{l}\right)$-we may assume without loss of generality that $\zeta_{l} \in k$. Then it follows from Lemma 2.5 (resp., Lemma 4.3(ii)) that $\operatorname{Im}\left(\rho_{0,4}^{\{l\}}\right)$ is topologically finitely generated (resp., pro- $l$ ). Therefore, it follows from $[22$, Lemma 2.8.7(c)] and [22, Corollary 2.8.5], together with the definition of the quotient $Q_{l}$, that condition (iii) is satisfied. This completes the proof of the above implication. 
DeFinition 7.5. We have the following.

(i) If $\mathfrak{p}$ is a nonarchimedean prime of $k$, then we write $v_{\mathfrak{p}}: k^{*} \rightarrow \mathbb{Z}$, where we write $k^{*}$ for the group of units of $k$ for the $\mathfrak{p}$-adic valuation such that if $p$ is the residue characteristic of $\mathfrak{p}$, then $v_{\mathfrak{p}}(p)$ coincides with the absolute ramification index of the completion of $k$ at $\mathfrak{p}$.

(ii) If $a$ is an element of $k^{*}$, then we write $[a]_{ \pm}$(resp., $\left.[a]_{+} ;[a]_{-}\right)$for the (necessarily finite) set of nonarchimedean primes $\mathfrak{p}$ of $k$ such that $v_{\mathfrak{p}}(a) \neq 0$ (resp., $\left.v_{\mathfrak{p}}(a)>0 ; v_{\mathfrak{p}}(a)<0\right)$.

LEMMA 7.6 (zeros and poles of certain divisors). We have the following:

(i) $[\lambda]_{+} \cap[\lambda]_{-}=\emptyset$;

(ii) $[\lambda]_{-}=[1-\lambda]_{-}$;

(iii) $[\lambda]_{+} \cap[1-\lambda]_{+}=\emptyset$;

(iv) $[\lambda]_{+} \neq \emptyset,[1-\lambda]_{+} \neq \emptyset$ if and only if $[\lambda]_{ \pm} \not\left[[1-\lambda]_{ \pm},[1-\lambda]_{ \pm} \nsubseteq[\lambda]_{ \pm}\right.$;

(v) suppose that

$$
\{\lambda, 1-\lambda, \lambda /(\lambda-1)\} \cap \mathfrak{o}_{k}^{*}=\emptyset,
$$

where we write $\mathfrak{o}_{k}^{*}$ for the group of units of $\mathfrak{o}_{k}$; then there exists an element $\lambda^{\prime}$ of

$$
\{\lambda, 1 / \lambda, 1-\lambda, 1 /(1-\lambda), \lambda /(\lambda-1),(\lambda-1) / \lambda\}
$$

such that $\left[\lambda^{\prime}\right]_{ \pm} \nsubseteq\left[1-\lambda^{\prime}\right]_{ \pm}$and $\left[1-\lambda^{\prime}\right]_{ \pm} \nsubseteq\left[\lambda^{\prime}\right]_{ \pm}$.

Proof. Assertion (i) follows from the definitions of $[-]_{+}$and $[-]_{-}$. Assertions (ii) and (iii) follow immediately from a straightforward calculation. Assertion (iv) follows immediately from assertions (i), (ii), and (iii). Finally, we verify assertion (v). Suppose that any element of $\mathfrak{m}_{\lambda} \stackrel{\text { def }}{=}\{\lambda, 1 / \lambda, 1-$ $\lambda, 1 /(1-\lambda), \lambda /(\lambda-1),(\lambda-1) / \lambda\}$ does not satisfy the desired condition. Now, since $\mathfrak{m}_{\lambda} \cap \mathfrak{o}_{k}^{*}=\emptyset$, any element $\lambda^{\prime} \in \mathfrak{m}_{\lambda}$ satisfies either $\left[\lambda^{\prime}\right]_{+} \neq \emptyset$ or $\left[1 / \lambda^{\prime}\right]_{+} \neq \emptyset$. Thus, it follows from assertion (iv) that, by replacing $\lambda$ with an element of $\mathfrak{m}_{\lambda}$, we may assume without loss of generality that

$$
\begin{gathered}
{[\lambda]_{+}=\emptyset ; \quad[1 / \lambda]_{+} \neq \emptyset ; \quad[(\lambda-1) / \lambda]_{+}=\emptyset ;} \\
{[\lambda /(\lambda-1)]_{+} \neq \emptyset ; \quad[1 /(1-\lambda)]_{+}=\emptyset ; \quad[1-\lambda]_{+} \neq \emptyset .}
\end{gathered}
$$

Therefore, it follows that $1 / \lambda, \lambda /(\lambda-1), 1-\lambda \in \mathfrak{o}_{k}$. In particular, we obtain that $\lambda /(\lambda-1) \in \mathfrak{o}_{k}^{*}$, in contradiction to the assumption that $\mathfrak{m}_{\lambda} \cap \mathfrak{o}_{k}^{*}=\emptyset$. This completes the proof of assertion (v). 
Definition 7.7. Let $l$ be an odd prime number. Then we say that $l$ satisfies the condition $\left(\dagger_{\lambda \in k}\right)$ if there exist two distinct nonarchimedean primes $\mathfrak{p}_{0}$ and $\mathfrak{q}_{0}$ of $k$ satisfying the following conditions:

(i) $\mathfrak{p}_{0} \notin \mathfrak{P}(k ; l), \mathfrak{p}_{0} \in[\lambda]_{ \pm}, \mathfrak{p}_{0} \notin[1-\lambda]_{ \pm}$, and $l$ is prime to $v_{\mathfrak{p}_{0}}(\lambda)$;

(ii) $\mathfrak{q}_{0} \notin \mathfrak{P}(k ; l), \mathfrak{q}_{0} \notin[\lambda]_{ \pm}, \mathfrak{q}_{0} \in[1-\lambda]_{ \pm}$, and $l$ is prime to $v_{\mathfrak{q}_{0}}(1-\lambda)$.

REMARK 7.7.1. It is easily verified that if $[\lambda]_{ \pm} \nsubseteq[1-\lambda]_{ \pm}$and $[1-$ $\lambda]_{ \pm} \nsubseteq[\lambda]_{ \pm}$, then there exists a cofinite set $\Sigma$ of prime numbers, that is, a (necessarily infinite) set of prime numbers obtained as the complement of a finite set of prime numbers in the set of all prime numbers such that if $l \in \Sigma$, then $l$ satisfies the condition $\left(\dagger_{\lambda \in k}\right)$.

LEMMA 7.8 (connectedness of a fiber). Let $l$ be an odd prime number, let $\zeta_{l} \in \bar{k}$ be a primitive lth root of unity, and let $\alpha_{l} \in \bar{k}$ (resp., $\beta_{l} \in \bar{k}$ ) be a solution of $t^{l}-\lambda$ (resp., $\left.t^{l}-(1-\lambda)\right)$, where $t$ is an indeterminate. Suppose that the prime number $l$ satisfies the condition $\left(\dagger_{\lambda \in k}\right)$. Then the following hold.

(i) The finite extension $k\left(\zeta_{l}, \alpha_{l}\right)$ (resp., $k\left(\zeta_{l}, \beta_{l}\right)$ ) of $k$ is ramified at $\mathfrak{p}_{0}$ (resp., $\mathfrak{q}_{0}$ ) (see Definition 7.7$)$ and unramified at $\mathfrak{q}_{0}$ (resp., $\mathfrak{p}_{0}$ ).

(ii) The extension $k_{l}\left(\alpha_{l}, \beta_{l}\right)$ of $k_{l}$ is of degree $l^{2}$.

(iii) The fiber of $X_{l} \otimes_{k} k_{l} \rightarrow \mathbb{P}_{k}^{1} \backslash\{0,1, \infty\}$ at the (image of the) $k$-rational point of $\mathbb{P}_{k}^{1} \backslash\{0,1, \infty\}$ corresponding to the element $\lambda \in k \backslash\{0,1\}$ is connected.

Proof. Assertion (i) follows from the definition of condition $\left(\dagger_{\lambda \in k}\right)$ and the fact that the degree of the finite extension $k\left(\zeta_{l}\right) / k$ is prime to $l$, together with, for example, [19, Chapter V, Lemma 3.3]. Assertion (ii) follows immediately from Lemma 7.2(i), together with assertion (i). Assertion (iii) follows immediately from assertion (ii), together with the fact that $\operatorname{Im}\left(\rho_{0,4}^{\{l\} \text {-geom }}\right)^{\mathrm{ab}} \otimes_{\mathbb{Z}_{l}} \mathbb{F}_{l}$ is of cardinality $l^{2}$.

THEOREM 7.9 (monodromic fullness of certain split hyperbolic curves of type $(0,4)$ over number fields). Let $l$ be an odd prime number, let $k$ be a number field (see the discussion on numbers in Section 0), and let $\lambda \in$ $k \backslash\{0,1\}$. Suppose that $l$ satisfies the condition $\left(\dagger_{\lambda \in k}\right)$ (see Definition 7.7). Then the hyperbolic curve $\left(\mathbb{P}_{k}^{1},\{0,1, \lambda, \infty\} \subseteq \mathbb{P}_{k}^{1}\right)$ of type $(0,4)$ over $k$ is $l$ monodromically full (see Definition 2.2(i)).

Proof. This follows from Lemmas 7.4 and 7.8(iii). 
Definition 7.10. Let $X$ be a hyperbolic curve of type $(0,4)$ over $k$. Then it follows immediately that there exists an element $\lambda_{X} \in \bar{k} \backslash\{0,1\}$ such that the hyperbolic curve $X \otimes_{k} \bar{k}$ is isomorphic over $\bar{k}$ to the hyperbolic curve

$$
\left(\mathbb{P}_{\bar{k}},\left\{0,1, \lambda_{X}, \infty\right\} \subseteq \mathbb{P}_{\bar{k}}\right)
$$

of type $(0,4)$ over $\bar{k}$. Now we write

$$
\mathfrak{m}_{X} \stackrel{\text { def }}{=}\left\{\lambda_{X}, 1 / \lambda_{X}, 1-\lambda_{X}, 1 /\left(1-\lambda_{X}\right), \lambda_{X} /\left(\lambda_{X}-1\right),\left(\lambda_{X}-1\right) / \lambda_{X}\right\} \subseteq \bar{k} .
$$

Note that it is well known that $\mathfrak{m}_{X}$ depends only on (and completely determines!) the isomorphism class of the hyperbolic curve $X \otimes_{k} \bar{k}$ over $\bar{k}$.

Corollary 7.11 (monodromic fullness of certain hyperbolic curves of type $(0,4)$ over number fields). Let $k$ be a number field (see the discussion on numbers in Section 0), let $\bar{k}$ be an algebraic closure of $k$, let $\mathfrak{o}_{\bar{k}}$ be the ring of integers of $\bar{k}$, let $\mathfrak{o}_{\bar{k}}^{*}$ be the group of units of $\mathfrak{o}_{\bar{k}}$, and let $X$ be a hyperbolic curve (see Definition 1.1(ii)) of type $(0,4)$ over $k$. If $\mathfrak{m}_{X} \cap \mathfrak{o}_{\bar{k}}^{*}=\emptyset$ (see Definition 7.10), then there exists a cofinite set $\Sigma$ of prime numbers, that is, a (necessarily infinite) set of prime numbers obtained as the complement of a finite set of prime numbers in the set of all prime numbers, such that the hyperbolic curve $X$ over $k$ is $\Sigma$-monodromically full (see Definition 2.2(i)).

In particular, if $\mathfrak{o}_{k}^{*} \subseteq k$ is the group of units of the ring of integers $\mathfrak{o}_{k}$ of $k$ and if $\lambda \in k \backslash\{0,1\}$ is an element of $k \backslash\{0,1\}$ such that

$$
\{\lambda, 1-\lambda, \lambda /(\lambda-1)\} \cap \mathfrak{o}_{k}^{*}=\emptyset
$$

then there exists a cofinite set $\Sigma$ of prime numbers such that the hyperbolic curve $\left(\mathbb{P}_{k}^{1},\{0,1, \lambda, \infty\} \subseteq \mathbb{P}_{k}^{1}\right)$ of type $(0,4)$ over $k$ is $\Sigma$-monodromically full.

Proof. This follows from Theorem 7.9, together with Lemma 7.6(v) and Remark 7.7.1.

Corollary 7.12 (monodromic fullness of split hyperbolic curves of type $(0,4)$ over the field of rational numbers or certain imaginary quadratic fields). Let $d$ be a square-free positive integer such that $d \neq 1,3$. Write $k$ for the field of rational numbers $\mathbb{Q}$ or the imaginary quadratic field $\mathbb{Q}(\sqrt{-d})$. If $\lambda \in k$, then the following conditions are equivalent:

(i) the hyperbolic curve $\left(\mathbb{P}_{k}^{1},\{0,1, \lambda, \infty\} \subseteq \mathbb{P}_{k}^{1}\right)$ - of type $(0,3)$ or $(0,4)$ over $k$ is not isomorphic to the hyperbolic curve $\left(\mathbb{P}_{k}^{1},\{0,1,-1, \infty\} \subseteq \mathbb{P}_{k}^{1}\right)$ of type $(0,4)$ over $k$; 
(ii) $\lambda$ is not equal to $-1,2,1 / 2$;

(iii) there exists a cofinite set $\Sigma$ of prime numbers, that is, a (necessarily infinite) set of prime numbers obtained as the complement of a finite set of prime numbers in the set of all prime numbers, such that the hyperbolic curve $\left(\mathbb{P}_{k}^{1},\{0,1, \lambda, \infty\} \subseteq \mathbb{P}_{k}^{1}\right)$-of type $(0,3)$ or $(0,4)$-over $k$ is $\Sigma$-monodromically full (see Definition 2.2(i));

(iv) there exists a prime number $l$ such that the hyperbolic curve $\left(\mathbb{P}_{k}^{1},\{0,1\right.$, $\left.\lambda, \infty\} \subseteq \mathbb{P}_{k}^{1}\right)$-of type $(0,3)$ or $(0,4)$-over $k$ is l-monodromically full.

Proof. The implication

$$
\text { (i) } \Longrightarrow(\text { ii) }
$$

is immediate. The implication

$$
\text { (ii) } \Longrightarrow(\text { iii) }
$$

follows from Corollary 7.11, together with the fact that the group of units of the ring of integers of $k$ is $\{ \pm 1\}$. The implication

$$
\text { (iii) } \Longrightarrow(\mathrm{iv})
$$

is immediate. Finally, we verify the implication

$$
\text { (iv) } \Longrightarrow(\mathrm{i}) \text {. }
$$

It is easily verified that $\mathbb{P}_{\mathbb{Q}}^{1} \backslash\{0,1,-1, \infty\}$ has some special symmetry (see Definition 3.3), that is, $\operatorname{Aut}_{\overline{\mathbb{Q}}}\left(\mathbb{P}_{\overline{\mathbb{Q}}} \backslash\{0,1,-1, \infty\}\right)$ is not isomorphic to $G_{0,4}$ (see Definition 3.1). Therefore, the above implication follows from Proposition 3.4 .

COROLlary 7.13 (Galois-theoretic characterization of isomorphism classes of certain hyperbolic curves of type $(0,4)$ over number fields). Let $k$ be a number field (see the discussion on numbers in Section 0), let $\bar{k}$ be an algebraic closure of $k$, let $\mathfrak{o}_{\bar{k}}$ be the ring of integers of $\bar{k}$, let $\mathfrak{o}_{\bar{k}}^{*}$ be the group of units of $\mathfrak{o}_{\bar{k}}$, let $G_{k} \stackrel{\text { def }}{=} \operatorname{Gal}(\bar{k} / k)$, and let $X_{1}=\left(C_{1}, D_{1} \subseteq C_{1}\right), X_{2}=$ $\left(C_{2}, D_{2} \subseteq C_{2}\right)$ be hyperbolic curves (see Definition 1.1(ii)) of type $(0,4)$ over $k$. Suppose that $\mathfrak{m}_{X_{1}} \cap \mathfrak{o}_{\bar{k}}^{*}=\mathfrak{m}_{X_{2}} \cap \mathfrak{o}_{\bar{k}}^{*}=\emptyset$ (see Definition 7.10). Then the following conditions are equivalent:

(i) $X_{1}$ is isomorphic to $X_{2}$ over $k$; 
(ii) there exists an infinite set $\Sigma$ of prime numbers such that, for any $l \in \Sigma$, if we write

$$
\rho_{X_{i} / k}^{\{l\}}: G_{k} \longrightarrow \operatorname{Out}\left(\pi_{1}\left(\left(C_{i} \backslash D_{i}\right) \otimes_{k} \bar{k}\right)^{(l)}\right)
$$

for the pro-l outer Galois representation associated to $X_{i}$, then $\operatorname{Ker}\left(\rho_{X_{1} / k}^{\{l\}}\right)=\operatorname{Ker}\left(\rho_{X_{2} / k}^{\{l\}}\right)$.

Proof. The implication

$$
\text { (i) } \Longrightarrow(\text { ii) }
$$

is immediate. On the other hand, the implication

$$
\text { (ii) } \Longrightarrow(\mathrm{i})
$$

follows immediately from Theorem 6.1, together with Corollary 7.11.

\section{§8. Example II: Nonisotrivial hyperbolic curves of type $(0,4)$}

In this section, we consider the monodromic fullness of nonisotrivial hyperbolic curves of type $(0,4)$.

Definition 8.1. Let $X$ be a hyperbolic curve over $k$. Then we say that $X$ is NF-isotrivial if there exist a finite extension $k^{\prime} \subseteq \bar{k}$ of $k$, a number field $k_{0} \subseteq k^{\prime}$ (see the discussion on numbers in Section 0) contained in $k^{\prime}$, and a hyperbolic curve $X_{0}$ over $k_{0}$ such that $X \otimes_{k} k^{\prime}$ is isomorphic to $X_{0} \otimes_{k_{0}} k^{\prime}$ over $k^{\prime}$ (see [26, Proposition 1.2(i)]).

COROllary 8.2 (monodromic fullness of nonisotrivial hyperbolic curves of type $(0,4))$. Let $k$ be a finitely generated field of characteristic zero (see the discussion on numbers in Section 0), and let $X$ be a hyperbolic curve (see Definition 1.1(ii)) of type $(0,4)$ over $k$ which is not $N F$-isotrivial (see Definition 8.1). Then there exists a cofinite set $\Sigma$ of prime numbers, that is, a (necessarily infinite) set of prime numbers obtained as the complement of a finite set of prime numbers in the set of all prime numbers, such that the hyperbolic curve $X$ over $k$ is $\Sigma$-monodromically full (see Definition 2.2(i)).

Proof. It is immediate that to verify Corollary 8.2 -by replacing $k$ with a suitable finite extension of $k$-we may assume without loss of generality that $X$ is split. Now, since $k$ is a finitely generated field of characteristic zero, there exist a subfield $k_{0} \subseteq k$ of $k$ and a scheme $V_{0}$ over $k_{0}$ satisfying the following conditions: 
(i) $k_{0}$ is a number field (see the discussion on numbers in Section 0);

(ii) $V_{0}$ is regular, separated, geometrically connected, and of finite type over $k_{0}$;

(iii) the function field of $V_{0}$ is isomorphic to $k$;

(iv) the split hyperbolic curve $X$ over $k$ extends to a split hyperbolic curve $X_{0}$ over $V_{0}$.

Now, since the natural outer homomorphism $\pi_{1}(\operatorname{Spec} k) \rightarrow \pi_{1}\left(V_{0}\right)$ (see (iii)) is surjective (see (ii)) and since the pro- $\Sigma$ outer monodromy representation $\rho_{X / k}^{\Sigma}$ factors through $\rho_{X_{0} / V_{0}}^{\Sigma}$ (see (iv)), it follows from the definition of the term monodromically full that, to verify Corollary 8.2, it suffices to show that there exists a closed point $v \in V_{0}$ of $V_{0}$ such that the hyperbolic curve $\left(X_{0}\right)_{v}$ over the residue field at $v \in V_{0}$ obtained as the fiber of the hyperbolic curve $X_{0}$ over $V_{0}$ at $v \in V_{0}$ is $\Sigma$-monodromically full for some cofinite set $\Sigma$ of prime numbers.

Write $\widetilde{s}_{X_{0} / V_{0}}: V_{0} \rightarrow \mathbb{P}_{k_{0}}^{1} \backslash\{0,1, \infty\}$ for the classifying morphism of the split hyperbolic curve $X_{0}$ over $V_{0}$ (see (iv), together with Lemma 4.1(i)). Then, since $X$ is not NF-isotrivial and since $\mathbb{P}_{k_{0}}^{1} \backslash\{0,1, \infty\}$ is of dimension one, it follows that the image of the morphism $\widetilde{s}_{X_{0} / V_{0}}$ is open. In particular, there exists a closed point $\bar{v}$ of $\mathbb{P}_{k_{0}}^{1} \backslash\{0,1, \infty\}$ contained in the image of $\widetilde{s}_{X_{0} / V_{0}}$ such that if $\lambda \in \bar{k}_{0} \backslash\{0,1\}$ is an element of $\bar{k}_{0} \backslash\{0,1\}$ naturally corresponding to $\bar{v} \in \mathbb{P}_{k_{0}}^{1} \backslash\{0,1, \infty\}$, then $\{\lambda, 1-\lambda, \lambda /(\lambda-1)\} \cap \mathfrak{o}_{\bar{k}_{0}}^{*}=\emptyset$, where $\bar{k}_{0}$ is an algebraic closure of $k_{0}, \mathfrak{o}_{\bar{k}_{0}}$ is the ring of integers of $\bar{k}_{0}$ (see (i)) and where $\mathfrak{o}_{\bar{k}_{0}}^{*}$ is the group of units of $\mathfrak{o}_{\bar{k}_{0}}$. Let $v \in V_{0}$ be a closed point of $V_{0}$ whose image via $\widetilde{s}_{X_{0} / V_{0}}$ is $\bar{v}$. Then it follows immediately from Corollary 7.11 that the hyperbolic curve $\left(X_{0}\right)_{v}$ over the residue field at $v \in V_{0}$ obtained as the fiber of the hyperbolic curve $X_{0}$ over $V_{0}$ at $v \in V_{0}$ is $\Sigma$-monodromically full for some cofinite set $\Sigma$ of prime numbers. This completes the proof of Corollary 8.2.

REMARK 8.2.1. It is immediate that Corollary 8.2 implies the following assertion. Let $k$ be a finitely generated field of characteristic zero (see the discussion on numbers in Section 0), and let $X$ be a hyperbolic curve (see Definition 1.1(ii)) of type $(0,4)$ over $k$. Suppose that there exists an infinite set $\Sigma$ of prime numbers such that if $l \in \Sigma$, then $X$ is not $l$-monodromically full (see Definition 2.2(i)). Then $X$ is NF-isotrivial (see Definition 8.1).

On the other hand, if in the above assertion one replaces $(0,4)$ with $(0, r)$ for some $r \geq 5$, then the conclusion no longer holds in general. A counterexample is as follows. Let $k_{0}$ be a number field, let $S \stackrel{\text { def }}{=} \mathbb{P}_{k_{0}}^{1} \backslash\{0,1,-1, \infty\}$, 
and let $k$ be the function field of $S$. Then the natural open immersion

$$
S \hookrightarrow \mathbb{P}_{k_{0}}^{1} \backslash\{0,1, \infty\}
$$

and the composite

$$
S \rightarrow \operatorname{Spec} k_{0} \hookrightarrow \mathbb{P}_{k_{0}}^{1} \backslash\{0,1, \infty\},
$$

where the first arrow is the structure morphism of $S$ and where the second arrow is the $k_{0}$-rational point corresponding to $-1 \in k_{0} \backslash\{0,1\}$, determine a morphism over $k$ from $S$ to the second configuration space of $\mathbb{P}_{k_{0}}^{1} \backslash\{0,1, \infty\}$. In particular, it follows immediately from Lemma 4.1(i) that we obtain a split hyperbolic curve $X$ over $k$ of type $(0,5)$. Now, since $X$ may be embedded as an open subscheme of $\mathbb{P}_{k}^{1} \backslash\{0,1,-1, \infty\}$, it follows immediately from Proposition 3.4 (see also the argument used in the proof of the implication (iv) $\Rightarrow$ (i) in the proof of Corollary 7.12), together with Remark 2.2.5, that, for any prime number $l$, the hyperbolic curve $X$ over $k$ is not $l$ monodromically full. On the other hand, it follows immediately from the definition of $X$ that $X$ is not NF-isotrivial.

COROLlary 8.3 (Galois-theoretic characterization of isomorphism classes of nonisotrivial hyperbolic curves of type $(0,4))$. Let $k$ be a finitely generated field of characteristic zero (see the discussion on numbers in Section 0), let $\bar{k}$ be an algebraic closure of $k$, let $G_{k} \stackrel{\text { def }}{=} \mathrm{Gal}(\bar{k} / k)$, and let $X_{1}=\left(C_{1}, D_{1} \subseteq C_{1}\right)$, $X_{2}=\left(C_{2}, D_{2} \subseteq C_{2}\right)$ be hyperbolic curves (see Definition 1.1(ii)) of type $(0,4)$ over $k$ which are not NF-isotrivial (see Definition 8.1). Then the following conditions are equivalent:

(i) $X_{1}$ is isomorphic to $X_{2}$ over $k$;

(ii) there exists an infinite set $\Sigma$ of prime numbers such that, for any $l \in \Sigma$, if we write

$$
\rho_{X_{i} / k}^{\{l\}}: G_{k} \longrightarrow \operatorname{Out}\left(\pi_{1}\left(\left(C_{i} \backslash D_{i}\right) \otimes_{k} \bar{k}\right)^{(l)}\right)
$$

for the pro-l outer Galois representation associated to $X_{i}$, then $\operatorname{Ker}\left(\rho_{X_{1} / k}^{\{l\}}\right)=\operatorname{Ker}\left(\rho_{X_{2} / k}^{\{l\}}\right)$.

Proof. The implication

$$
\text { (i) } \Longrightarrow(\text { ii) }
$$

is immediate. On the other hand, the implication

$$
\text { (ii) } \Longrightarrow(\text { i) }
$$

follows immediately from Theorem 6.1, together with Corollary 8.2. 


\section{Appendix. Ramification of outer Galois representations and iso- morphism classes of hyperbolic curves}

In this appendix, we prove finiteness results, which are related to the main result of this paper (see Theorem A.3 and Corollary A.4 below). It seems to the author that the results appearing in this section are likely to be well known; however, since the results could not be found in the literature, we decided to give proofs of the results in this section. Here, let $l$ be a prime number, let $k$ be a number field (see the discussion on numbers in Section 0), and let $(g, r)$ be a pair of nonnegative integers such that $2 g-2+r>0$.

Definition A.1. Let $N \subseteq G_{k}$ be a normal closed subgroup of $G_{k}$, and let $\mathfrak{P}$ be a set of primes of $k$. Then we write

$$
\mathcal{I}^{\mathrm{Gal}}(l, k, g, r, N)\left(\operatorname{resp} ., \mathcal{I}^{\mathrm{unr}}(l, k, g, r, \mathfrak{P})\right)
$$

for the set of the isomorphism classes over $k$ of hyperbolic curves $X=$ $(C, D \subseteq C)$ of type $(g, r)$ over $k$ satisfying the following condition. If we write

$$
\rho_{X / k}^{\{l\}}: G_{k} \longrightarrow \operatorname{Out}\left(\pi_{1}\left((C \backslash D) \otimes_{k} \bar{k}\right)^{(l)}\right)
$$

for the pro- $l$ outer Galois representation associated to $X$, then the kernel of $\rho_{X / k}^{\{l\}}$ coincides with $N \subseteq G_{k}$ (resp., then $\rho_{X / k}^{\{l\}}$ is unramified outside $\mathfrak{P}$ ).

REMARK A.2. If $N \subseteq G_{k}$ is a normal closed subgroup of $G_{k}$ obtained as the kernel of the pro- $l$ outer Galois representation associated to a hyperbolic curve over $k$, then it is easily verified that there exists a finite set $\mathfrak{P}$ of primes of $k$ such that $\mathcal{I}^{\mathrm{Gal}}(l, k, g, r, N) \subseteq \mathcal{I}^{\mathrm{unr}}(l, k, g, r, \mathfrak{P})$.

The main purpose of this section is to prove the following fact.

THEOREM A.3. Let $l$ be a prime number, let $k$ be a number field (see the discussion on numbers in Section 0), let $\bar{k}$ be an algebraic closure of $k$, let $G_{k} \stackrel{\text { def }}{=} \operatorname{Gal}(\bar{k} / k)$, let $(g, r)$ be a pair of nonnegative integers such that $2 g-2+$ $r>0$, and let $\mathfrak{P}$ be a finite set of primes of $k$. Then the set $\mathcal{I}^{\mathrm{unr}}(l, k, g, r, \mathfrak{P})$ (see Definition A.1) is finite.

By Theorem A.3, together with Remark A.2, we obtain the following corollary.

Corollary A.4. Let $l$ be a prime number, let $k$ be a number field (see the discussion on numbers in Section 0), let $\bar{k}$ be an algebraic closure of 
$k$, let $G_{k} \stackrel{\text { def }}{=} \operatorname{Gal}(\bar{k} / k)$, let $(g, r)$ be a pair of nonnegative integers such that $2 g-2+r>0$, and let $N \subseteq G_{k}$ be a normal closed subgroup of $G_{k}$. Then the set $\mathcal{I}^{\mathrm{Gal}}(l, k, g, r, N)$ (see Definition A.1) is finite.

The rest of the appendix is devoted to proving Theorem A.3.

Lemma A.5. Let $\mathfrak{P}$ be a finite set of primes of $k$, and let $X=(C, D \subseteq$ $C)$ be a hyperbolic curve over $k$ whose isomorphism class over $k$ is in $\mathcal{I}^{\text {unr }}(l, k, g, r, \mathfrak{P})$. Then there exists a finite extension $k(l, k, r, \mathfrak{P}) \subseteq \bar{k}$ of $k$ that depends only on $l, k, r$, and $\mathfrak{P}$ such that the hyperbolic curve $X \otimes_{k}$ $k(l, k, r, \mathfrak{P})$ over $k(l, k, r, \mathfrak{P})$ is split (see Definition 1.5(i)).

Proof. To prove Lemma A.5-by replacing $\mathfrak{P}$ with a finite set of primes of $k$ containing $\mathfrak{P}$ and the set of the nonarchimedean primes $\mathfrak{p}$ of $k$ such that the residue characteristic of $\mathfrak{p}$ is $l$ - we may assume without loss of generality that every nonarchimedean prime of $k$ whose residue characteristic is $l$ is contained in $\mathfrak{P}$. Then it follows immediately from Oda-Tamagawa's criterion for good reduction of hyperbolic curves (see [25, Theorem 0.8]) that any irreducible component of $D$ is isomorphic to the spectrum of a finite extension of $k$ which is unramified outside $\mathfrak{P}$. On the other hand, it follows immediately from a well-known theorem of Hermite and Minkowski that there are only finitely many isomorphism classes of finite extensions of $k$ of degree at most $r$ which are unramified outside $\mathfrak{P}$. Therefore, if we write $k(l, k, r, \mathfrak{P})$ for the composite field of all extension fields (in $\bar{k}$ ) of degree at most $r$ which are unramified outside $\mathfrak{P}$, then $k(l, k, r, \mathfrak{P})$ satisfies the desired condition. This completes the proof of Lemma A.5.

LEMMA A.6. Let $k^{\prime}$ be a finite extension of $k$, and let $Y$ be a hyperbolic curve over $k^{\prime}$. Then there are only finitely many isomorphism classes over $k$ of hyperbolic curves $X$ over $k$ satisfying the following condition: $X \otimes_{k} k^{\prime}$ is isomorphic to $Y$ over $k^{\prime}$.

Proof. To verify Lemma A.6-by replacing $k^{\prime}$ with a finite extension of $k^{\prime}$-we may assume without loss of generality that the extension $k^{\prime}$ of $k$ is Galois. Write $\mathcal{D}$ for the set of the isomorphism classes $\left[X, \phi: X \otimes_{k} k^{\prime} \stackrel{\sim}{\rightarrow} Y\right]$ of pairs $\left(X, \phi: X \otimes_{k} k^{\prime} \stackrel{\sim}{\rightarrow} Y\right)$ of hyperbolic curves $X$ over $k$ and isomorphisms $\phi: X \otimes_{k} k^{\prime} \stackrel{\sim}{\rightarrow} Y$ over $k^{\prime}$, where we say that a pair $\left(X_{1}, \phi_{1}: X_{1} \otimes_{k} k^{\prime} \stackrel{\sim}{\rightarrow} Y\right)$ is isomorphic to a pair $\left(X_{2}, \phi_{2}: X_{2} \otimes_{k} k^{\prime} \stackrel{\sim}{\rightarrow} Y\right)$ if there exists an isomorphism $\psi: X_{1} \stackrel{\sim}{\rightarrow} X_{2}$ over $k$ such that $\phi_{2} \circ \psi=\phi_{1}$. To verify Lemma A.6, it is clear that it suffices to show that this set $\mathcal{D}$ is finite. Moreover, to verify 
the finiteness of $\mathcal{D}$, it is clear that we may assume without loss of generality that $\mathcal{D}$ is nonempty. Let us fix an element $\left[X_{0}, \phi_{0}: X_{0} \otimes_{k} k^{\prime} \stackrel{\sim}{\rightarrow} Y\right] \in \mathcal{D}$ of $\mathcal{D}$. Then we obtain a map

$$
\begin{aligned}
\mathcal{D} & \longrightarrow Z^{1}\left(\operatorname{Gal}\left(k^{\prime} / k\right), \operatorname{Aut}_{k^{\prime}}(Y)\right) \\
{\left[X, \phi: X \otimes_{k} k^{\prime} \stackrel{\sim}{\rightarrow} Y\right] } & \mapsto\left(g \mapsto \phi \circ g^{-1} \circ \phi^{-1} \circ \phi_{0} \circ g \circ \phi_{0}^{-1}\right),
\end{aligned}
$$

where the action of $\operatorname{Gal}\left(k^{\prime} / k\right)$ on $\operatorname{Aut}_{k^{\prime}}(Y)$ is given by

$$
\begin{aligned}
\operatorname{Gal}\left(k^{\prime} / k\right) & \longrightarrow \operatorname{Aut}\left(\operatorname{Aut}_{k^{\prime}}(Y)\right) \\
g & \mapsto\left(f \mapsto \phi_{0} \circ g^{-1} \circ \phi_{0}^{-1} \circ f \circ \phi_{0} \circ g \circ \phi_{0}^{-1}\right) .
\end{aligned}
$$

Moreover, by Galois descent, this map is injective. Therefore, the finiteness of $\mathcal{D}$ follows from the finiteness of $\operatorname{Gal}\left(k^{\prime} / k\right)$ and $\operatorname{Aut}_{k^{\prime}}(Y)$.

Proof of Theorem A.3. To prove Theorem A.3-by replacing $\mathfrak{P}$ with a finite set of primes of $k$ containing $\mathfrak{P}$ and the set of the nonarchimedean primes $\mathfrak{p}$ of $k$ such that the residue characteristic of $\mathfrak{p}$ is $l$-we may assume without loss of generality that every nonarchimedean prime of $k$ whose residue characteristic is $l$ is contained in $\mathfrak{P}$. Moreover, it follows from Lemma A.6 that to prove Theorem A.3, it suffices to verify that if we write $\mathcal{I}_{1} \subseteq \mathcal{I}^{\text {unr }}(l, k, g, r, \mathfrak{P})$ for the subset of $\mathcal{I}^{\text {unr }}(l, k, g, r, \mathfrak{P})$ consisting of the isomorphism classes over $k$ of hyperbolic curves which are split, then $\mathcal{I}_{1}$ is finite. Now if $X=(C, D \subseteq C)$ is a hyperbolic curve over $k$ whose isomorphism class over $k$ is in $\mathcal{I}_{1}$, then it follows from Oda-Tamagawa's criterion for good reduction of hyperbolic curves (see [25, Theorem 0.8]) that the proper curve $C$ admits good reduction at all primes outside $\mathfrak{P}$. Therefore, if $g \geq 1$ (resp., if $g=0$ ), then it follows from a well-known theorem of Faltings and Shafarevich (resp., the fact that $X$ is split) that the set consisting of the isomorphism classes over $k$ of the proper curves $C$ appearing in the elements $[C, D \subseteq C]$ of $\mathcal{I}_{1}$ is finite. Thus, to prove Theorem A.3, it suffices to verify that, for a hyperbolic curve $X_{0}=\left(C_{0}, D_{0} \subseteq C_{0}\right)$ over $k$ whose isomorphism class over $k$ is in $\mathcal{I}_{1}$, if we write $\mathcal{I}_{2} \subseteq \mathcal{I}_{1}$ for the subset of $\mathcal{I}_{1}$ consisting of the isomorphism classes over $k$ of hyperbolic curves $X=(C, D \subseteq C)$ over $k$ such that the proper curves $C$ are isomorphic to the proper curve $C_{0}$ over $k$, then $\mathcal{I}_{2}$ is finite. On the other hand, this follows immediately from two wellknown theorems of Mahler and Siegel and Faltings and Mordell, together with Oda-Tamagawa's criterion for good reduction of hyperbolic curves (see [25, Theorem 0.8]). This completes the proof of Theorem A.3. 
Acknowledgments. Part of this research was carried out during 2009 while the author was a visiting fellow in the "Non-Abelian Fundamental Groups in Arithmetic Geometry (NAG)" program at the Isaac Newton Institute for Mathematical Sciences of the University of Cambridge, and the author thanks the institute and the organizers of the NAG program for inviting me and for their hospitality during my stay. I also thank Professor Shinichi Mochizuki and the referees for helpful comments.

\section{References}

[1] M. P. Anderson, Exactness properties of profinite completion functors, Topology 13 (1974), 229-239.

[2] G. Anderson and Y. Ihara, Pro-l branched coverings of $\mathbb{P}^{1}$ and higher circular l-units, Ann. of Math. (2) 128 (1988), 271-293.

[3] M. Asada, The faithfulness of the monodromy representations associated with certain families of algebraic curves, J. Pure Appl. Algebra 159 (2001), 123-147.

[4] C. L. Chai and F. Oort, A note on the existence of absolutely simple Jacobians, J. Pure Appl. Algebra 155 (2001), 115-120.

[5] P. Deligne and D. Mumford, The irreducibility of the space of curves of given genus, Publ. Math. Inst. Hautes Études Sci. 36 (1969), 75-109.

[6] A. Grothendieck and J. Dieudonné, Éléments de géométrie algébrique, III: Étude cohomologique des faisceaux cohérents, I, Publ. Math. Inst. Hautes Études Sci. 11 (1961).

[7] —— Groupes de monodromie en géométrie algébrique, I, Séminaire de Géométrie Algébrique du Bois-Marie (SGA 7 I), Lecture Notes in Math. 288, Springer, Berlin, 1972.

[8] Y. Hoshi and S. Mochizuki, On the combinatorial anabelian geometry of nodally nondegenerate outer representations, preprint, to appear in Hiroshima Math. J.

[9] Y. Ihara and H. Nakamura, On deformation of maximally degenerate stable marked curves and Oda's problem, J. Reine Angew. Math. 487 (1997), 125-151.

[10] F. Knudsen, The projectivity of the moduli space of stable curves, II: The stacks $\mathcal{M}_{g, n}$, Math. Scand. 52 (1983), 161-199.

[11] M. Matsumoto and A. Tamagawa, Mapping-class-group action versus Galois action on profinite fundamental groups, Amer. J. Math. 122 (2000), 1017-1026.

[12] S. Mochizuki, Correspondences on hyperbolic curves, J. Pure Appl. Algebra 131 (1998), 227-244.

[13] - The local pro-p anabelian geometry of curves, Invent. Math. 138 (1999), $319-423$.

[14] - "Topics surrounding the anabelian geometry of hyperbolic curves" in Galois Groups and Fundamental Groups, Math. Sci. Res. Inst. Publ. 41, Cambridge University Press, Cambridge, 2003, 119-165.

[15] - "The absolute anabelian geometry of hyperbolic curves" in Galois Theory and Modular Forms, Dev. Math. 11, Kluwer, Boston, 2004, 77-122.

[16] S. Mochizuki and A. Tamagawa, The algebraic and anabelian geometry of configuration spaces, Hokkaido Math. J. 37 (2008), 75-131.

[17] H. Nakamura, Galois rigidity of pure sphere braid groups and profinite calculus, J. Math. Sci. Univ. Tokyo 1 (1994), 71-136. 
[18] H. Nakamura, N. Takao, and R. Ueno, Some stability properties of Teichmüller modular function fields with pro-l weight structures, Math. Ann. 302 (1995), 197-213.

[19] J. Neukirch, Algebraic Number Theory, Grundlehren Math. Wiss. 322, Springer, Berlin, 1999.

[20] T. Oda, "Étale homotopy type of the moduli spaces of algebraic curves" in Geometric Galois Actions, Vol. 1, London Math. Soc. Lecture Note Ser. 242, Cambridge University Press, Cambridge, 1997, 85-95.

[21] B. Poonen, Varieties without extra automorphisms, II: Hyperelliptic curves, Math. Res. Lett. 7 (2000), 77-82.

[22] L. Ribes and P. Zalesskii, Profinite Groups, Ergeb. Math. Grenzgeb. (3) 40, Springer, Berlin, 2000.

[23] J. P. Serre and J. Tate, Good reduction of abelian varieties, Ann. of Math. (2) 88 (1968), 492-517.

[24] N. Takao, Braid monodromies on proper curves and pro-l Galois representations, preprint, to appear in J. Inst. Math. Jussieu.

[25] A. Tamagawa, The Grothendieck conjecture for affine curves, Compos. Math. 109 (1997), 135-194.

[26] $\longrightarrow$ Resolution of nonsingularities of families of curves, Publ. Res. Inst. Math. Sci. 40 (2004), 1291-1336.

Research Institute for Mathematical Sciences

Kyoto University

Kyoto 606-8502

Japan

yuichiro@kurims.kyoto-u.ac.jp 